\title{
Multibody decay analyses: A new phenomenological model for meson-meson subamplitudes
}

\author{
P. C. Magalhães $\oplus^{*}$ \\ H.H. Wills Physics Laboratory, University of Bristol, Bristol BS8 1TLB, United Kingdom
}

A. C. $\operatorname{dos}$ Reis $\odot$

Centro Brasileiro de Pesquisas Fisicas, 22.290-180 Rio de Janeiro, Brazil

M. R. Robilotta $\odot$

Instituto de Fsica, Universidade de São Paulo, 05508-090 São Paulo, Brazil

(Received 23 July 2020; accepted 1 September 2020; published 19 October 2020)

\begin{abstract}
Meson-meson amplitudes are important on their own and also play key roles in analyses of heavy-meson and tau decays. In this work we propose a new phenomenological model suited to all $S U(3)$ mesonic twobody final-state interactions up to energies around $2 \mathrm{GeV}$. It is aimed at replacing those entering the old isobar model, produced in the 1960s, long before the development of QCD. The only similarity between our new proposal and amplitudes used in the isobar model concerns vector resonances in the elastic regime. In other situations, especially those involving scalar resonances and coupled channels, the isobar model is not compatible with post-QCD dynamics. In order to support these claims convincingly and to motivate our approach, we consider applications to the $\pi \pi$ amplitude and compare our version with the isobar model in several different instances. We also show that the new model provides a clear indication of the mechanism responsible for the sharp rise observed in the $\pi \pi$ phase around $1 \mathrm{GeV}$. The phenomenological amplitudes proposed here are suited to any number of resonances in a given channel and rely just on masses and coupling constants as free parameters. Concerning theory, they incorporate chiral symmetry at low energies, include coupled channels, and respect unitarity whenever appropriate.
\end{abstract}

DOI: $10.1103 /$ PhysRevD.102.076012

\section{MOTIVATION}

In the last decade, a considerable amount of precise data has been produced from BABAR, Belle, BES, LHCb experiments on nonleptonic three-body decays of $D$ and $B$ mesons as well as on tau decays into pseudoscalars. More comprehensive investigations can be done nowadays, using the very large and pure samples provided by the LHC experiments, and still more data is expected in the near future, including neutral particles, with Belle II, BES III, and LHCb (Run 2) experiments.

These decays involve two distinct sets of interactions. They begin with a primary vertex, in which the light $S U(3)$ quarks produced in the weak reaction disturb the surrounding QCD vacuum and give rise to an initial set of mesons.

\footnotetext{
* Corresponding author. p.magalhaes@bristol.ac.uk

Published by the American Physical Society under the terms of the Creative Commons Attribution 4.0 International license. Further distribution of this work must maintain attribution to the author(s) and the published article's title, journal citation, and DOI. Funded by SCOAP ${ }^{3}$.
}

This state then evolves by means of purely hadronic final-state interactions (FSIs), whereby mesons rescatter many times before being detected. This rich hadronic finalstate structure is an important source of spectroscopic information about resonances and we recall that the existence of the controversial scalar states $f_{0}(500)$ [1] and $K_{0}^{*}(700)$ [2] was confirmed in three-body decays. Final-state interactions are also relevant in the study of $C P$ violation [3].

The analysis of nonleptonic three-body heavy-meson decays is technically involved and relies on models. The standard isobar model (SIM) is by far the most popular choice among phenomenologists interested in resonance parameters. It was proposed in the early 1960s, long before the development of QCD, and fails to incorporate the new understanding of quark dynamics produced by the theory. Its basic assumption is that a decay amplitude can be represented by a coherent sum of both nonresonant and resonant contributions, with emphasis on the latter. The amplitude for the decay $H(Q) \rightarrow P_{a}\left(q_{a}\right) P_{b}\left(q_{b}\right) P_{c}\left(q_{c}\right)$ of a heavy meson $H$ into three pseudoscalars $P$ is denoted by $\mathcal{T}$ and depends on the invariant masses $m_{a b}^{2}=\left(q_{a}-q_{b}\right)^{2}$ and $m_{a c}^{2}=\left(q_{a}-q_{c}\right)^{2}$. 
What we define as the standard isobar model assumes that $\mathcal{T}$ can be written as

$$
\begin{aligned}
\mathcal{T}\left(m_{a b}^{2}, m_{a c}^{2}\right)= & c_{n r} \tau_{n r}\left(m_{a b}^{2}, m_{a c}^{2}\right) \\
& +\left[\sum_{k} c_{k} \tau_{k}\left(m_{a b}^{2}\right)+\sum_{j} c_{j} \tau_{j}\left(m_{a c}^{2}\right)\right],
\end{aligned}
$$

where $k$ and $j$ are resonance labels that can be the same for a symmetric decay. The first term in Eq. (1) is nonresonant and that within square brackets implements the quasi-twobody or $(2+1)$ approximation, in which only the interactions of a pair of particles matter and the third one the bachelor is just a spectator. The $\tau_{k}(s)$ functions, for $s=m_{a b}^{2}, m_{a c}^{2}$, represent dynamic two-body amplitudes and the complex coefficients $c_{k}=e^{i \theta_{k}}$ are fitting parameters. In the absence of a theory, the first term is usually taken to be $\tau_{n r}=1$. For each resonance considered, one uses $\tau_{k}=[\mathrm{FF}] \times[$ angular factor $] \times[\text { line shape }]_{k}$, where [FF] stands for form factors, [angular factor] is associated with spin, and [line shape $]_{k}$ represents a Breit-Wigner (BW) function depending on a mass $m_{k}$ and a width $\Gamma_{k}$, given by

$$
[\text { line shape }]_{k} \rightarrow[\mathrm{BW}]_{k}=\frac{1}{\left[s-m_{k}^{2}+i m_{k} \Gamma_{k}\right]} .
$$

For some states, variations such as the Flatté and GounarisSakurai representations are used. In applications, both the qualities and quantities of resonances employed are regulated $a d h o c$ and the outcome of isobar model analyses are values for masses, widths, fit fractions, and sometimes mixing couplings. In particular, fit fractions are associated with the complex parameters $c_{n r}$ and $c_{k}$, which are neither directly related to an underlying dynamics nor allow the identification of substructures. Important limitations of the isobar model are presented below.

(1) Even if one overlooks the problem of ascribing physical meanings to parameters extracted from the isobar model, there is another issue. Strictly speaking, their numerical values depend on the particular assumptions underlying how Eq. (1) is used, namely, the nonresonant term and the number and isospins of resonances employed. Therefore, the numerical meaning of the parameters extracted always remains attached to the specific reaction employed to derive them. Final-state interactions incorporated into the decay amplitude $\mathcal{T}$ include both proper three-body interactions and a wide range of elastic and inelastic two-body subamplitudes $\mathcal{A}$ involving resonances and coupled channels, as we review in Sec. II. In a given decay, the main information about resonances is codified in the $\mathcal{A}$ 's and, even if there are exceptions, it is important to distinguish them from $\mathcal{T}$. A conspicuous difference between these amplitudes is that the latter includes weak vertices and the former does not, but this is sometimes bypassed in the literature. For instance, there is no justification for the assumption that the $\mathcal{A}$ 's are either identical or proportional to $\mathcal{T}$, as found in a partial-wave analysis of the $S$-wave $K^{-} \pi^{+}$amplitude from the decay $D^{+} \rightarrow K^{-} \pi^{+} \pi^{+}$produced some time ago [2]. As a matter of fact, the empirical phase is different from that produced by LASS for $K \pi$ scattering data [4]. As expected, this discrepancy arose because one was comparing different objects and was later explained by considering meson loops in the weak sector of $\mathcal{T}$ [5-8].

(2) The extraction of information from the isobar model is hampered by the presence of nonresonant terms. An important message brought to hadron physics by QCD is that, provided enough energy is available, the light-quark condensate does show up and several pseudoscalars can be produced in a single vertex. For instance, the process $e^{-} e^{+} \rightarrow 4 \pi$ involves the multimeson matrix element $\left\langle\pi \pi \pi \pi\left|J_{\gamma}^{\mu}\right| 0\right\rangle$, where $J_{\gamma}^{\mu}$ is the electromagnetic current [9]. A similar matrix element, with the weak current $(V-A)^{\mu}$, describes the decay $\tau \rightarrow \nu 4 \pi$ [9]. In a recent work [10], we studied the doubly Cabibbosuppressed decay $D^{+} \rightarrow K^{-} K^{+} K^{+}$departing from a nonresonant term based on the axial-current matrix element $\left\langle K^{-} K^{+} K^{+}\left|A^{\mu}\right| 0\right\rangle$, describing the annihilation of the $D^{+}$into a $W^{+}$which subsequently hadronizes. In that case, nonresonant terms and those involving resonances are entangled by a kind of diagrammatic continuity.

(3) In principle, the functions $\tau_{k}(s)$ in Eq. (1) do contain information about two-body interactions, but extracting it is difficult, for isospin channels are not clearly identified. Scattering amplitudes $\mathcal{A}$ depend on both the angular momentum $J$ and the isospin $I$ of the channel considered, whereas just a $J$ dependence can be extracted from an empirical decay amplitude $\tau_{k}$. Therefore, an attempt to extract $\mathcal{A}^{(J, I)}$ from $\tau_{k}^{(J)}$ would amount to an artificial generation of physical content from the reaction considered.

(4) For processes requiring several resonances with the same quantum numbers, SIM amplitudes given by sums $\Sigma c_{k} \tau_{k}$ violate unitarity, a criticism raised by many authors [11-13]. At present, there are solid conceptual techniques aimed at preserving unitarity in amplitudes involving several resonances [14], as discussed in Sec. II. Thus, nowadays, it is difficult to justify the use of problematic guess functions based on sums of individual line shapes given by Eq. (2).

(5) Meson-meson isoscalar amplitudes $\mathcal{A}$ include important inelasticities due to couplings of intermediate states. For instance, in $\pi \pi$ scattering the $K \bar{K}$ inelastic channel [15] opens at $E \simeq 1 \mathrm{GeV}$. So, this energy represents the upper bound for the validity of 
Eq. (1), since there is no room in the BW-like representation of functions $\tau_{k}$ [Eq. (2)] for the incorporation of coupled channels. In general, guess functions better suited for accommodating data should have structures similar to those used in meson-meson scattering [15-17]. In the SIM, the guess functions that are usually employed are not suited to accommodate coupled channels. The role of resonances above inelastic thresholds is discussed in Sec. V.

All of the problems of the standard isobar model mentioned above tend to corrode the physical meaning of the parameters it yields from fits. Since it was proposed more than half a century ago many of the limitations pointed out above were understood and tamed, especially owing to the formulation of QCD. As a consequence, nowadays, serious flaws of the model are already rather clear, such as that it violates unitarity, does not incorporate isospin, and especially important is totally unsuited for dealing with coupled channels. In the $S U(3)$ sector, scattering amplitudes for pions, kaons, and etas are strongly coupled and cannot be represented as sums of individual contributions. At present, as is well known, QCD cannot be directly applied to heavy meson decays, but their effective counterparts can. Effective Lagrangians rely only on hadron masses and coupling constants, ensuring that the physical meaning of parameters is preserved from process to process. Thus, guess functions for fitting heavy-meson decay data departing from Lagrangians deal with the same free parameters as employed in scattering amplitudes. This makes the mutual comparison of their values meaningful.

This work is part of a program aimed at constructing guess functions for heavy-meson decays departing from effective Lagrangians. Here, we concentrate on the twobody scattering amplitudes $\mathcal{A}$, which are directly associated with observed quantities and also important substructures of decay amplitudes. We depart from a previous work on $D^{+} \rightarrow K^{-} K^{+} K^{+}$where a three-body amplitude was constructed based on effective Lagrangians with chiral symmetry and contained unitarized scattering subamplitudes [10]. Although fits to Dalitz plot data were better than those based on the standard isobar model [18], that work was performed in the $K$-matrix approximation. We draw attention to the fact that this $K$-matrix approximation is not the same thing as the K-Matrix approach [19] used in some amplitude analyses. Here, we propose a model which allows one to go beyond this approximation and discuss its implications.

Our presentation is organized as follows. In Sec. II we review how heavy-meson decay amplitudes are related to weak vertices, scattering amplitudes, and form factors. This is intended to provide a broad conceptual framework for criticisms of the isobar model. The full scattering amplitudes for the $S U(3)$ pseudoscalars in the coupled channel formalism are presented in the Appendix C, combining interaction kernels and two-meson propagators given in Appendices A and B. In Sec. III we present the full scattering amplitudes and specialize to the $\pi \pi$ amplitude, which is used as a standard for assessing the limitations of the isobar model. In Sec. IV we discuss these limitations regarding post-QCD physics and unitarity. In Sec. V we discuss the impact of coupled channels on the problem and show that the meaning of a resonance as an independent contribution is lost in the inelastic region, supporting our claim that BW line shapes should not be used above $1 \mathrm{GeV}$. We also compare coupled and uncoupled amplitudes and show that the impact of coupling is huge. In Sec. VI we present our model for the real part of two-meson propagators which allows one to go beyond the $K$-matrix approximation. In Sec. VII we add an extra resonance to each scalar channel using the methodology we developed and show the potentiality of our model for extensions to higher energies. Finally, in Sec. VIII we summarize our conclusions.

\section{SCHEMATIC DYNAMICS}

The theoretical description of a heavy-meson $H$ decaying into three light pseudoscalars $P_{a} P_{b} P_{c}$ involves several classes of entangled problems and is necessarily rather complicated. Below, we use simple topological arguments, based on hadronic degrees of freedom, to classify these problems. We rely on building blocks determined by proper hadronic interactions, defined as those associated with diagrams that cannot be separated into two pieces by cutting hadron lines only. As one is dealing with weak and strong interactions simultaneously, it is convenient to isolate these two sectors as much as possible.

The basic weak interactions producing the decay of a heavy meson involve quarks in the QCD vacuum and were classified by Chau [20]. At the hadronic level, the primary weak vertex contains two kinds of proper Feynman diagrams (shown in Fig. 1) describing the processes $H \rightarrow$ $P_{a} P_{b} P_{c}$ and $H \rightarrow P_{a} R_{x}$, where $R_{x}$ is a light resonance which later decays as $R_{x} \rightarrow P_{b} P_{c}$. At this stage, this resonance is described by a bare pole and does not have a width yet. The green blob does not include hadronic degrees of freedom, but can contain strong processes in the form of quarks and gluon exchanges. In the literature the primary vertex is described by means of either factorization techniques [21] or effective Lagrangians [22].

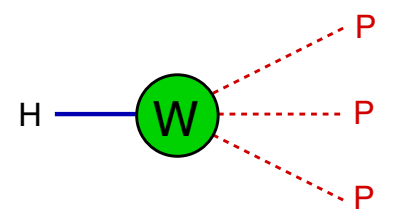

(a)

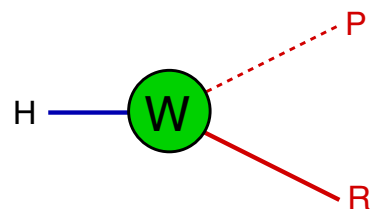

(b)
FIG. 1. Contributions to the primary weak vertex: (a) $H \rightarrow$ $P_{a} P_{b} P_{c}$ and (b) $H \rightarrow P_{a} R_{x}$. 
The mesons produced in diagram (a) of Fig. 1 can go directly to the detector and give rise to a nonresonant contribution. Alternatively, it is possible that the hadrons produced in diagrams (a) and (b) of Fig. 1 and 1(b) have various forms of strong interactions before reaching the detector. In this case, one talks about FSIs, which are necessarily strong.

Nowadays, most approaches tend to organize the FSIs departing from chiral perturbation theory (ChPT). Although lattice QCD is improving [23], ChPT still is the best available effective representation of QCD at low energies [24-26] and can accommodate resonances [27]. As resonances correspond to nonperturbative states, predictions from ChPT are precise up to energies below the $\rho(770)$ mass. Beyond that point, one has to resort to extensions of ChPT, which may be performed by means of either dynamical models $[10,16,19,28,29]$ or dispersion relations $[17,30]$. Here we describe the basics of the former approach, which we find more suited to phenomenological studies of problems involving several resonances. The idea is to define a few basic building blocks, as displayed in Fig. 2, and to construct all relevant interactions departing from them. The diagram in diagram (a) of Fig. 2 represents a four-meson contact interaction, predicted by ChPT to be the single leading contribution at low energies and corresponds to an amplitude given by a second-order polynomial in momenta and meson masses. The process in diagram (c) of Fig. 2 is a higher-order term, describing a proper sixmeson vertex. Resonances are also included in the chiral formalism [27] and the diagrams (b) and (d) of Fig. 2 are associated with their decay and scattering amplitudes. To our knowledge, the diagram (c) in Fig. 2 has not yet been included in realistic calculations of heavy-meson decays, whereas interactions described by diagram (d) of Fig. 2 were considered in a phenomenological description of the process $\sigma \sigma(\rho \rho) \rightarrow 4 \pi$ contributing to $\pi \pi$ scattering [29].

The diagrams of Fig. 2 resemble interaction potentials $V$ in quantum mechanics and, to determine the full solution of a problem, one has to solve a dynamical equation analogous to that of the Lippmann-Schwinger equation. This is not feasible in field theory and one has to resort to a piecemeal evaluation of perturbative corrections. The procedure is similar to that used in quantum mechanics, where full and free solutions are related by a series of the form $1+g V+g V g V+\cdots$, where $g$ is the free propagator. In the present problem, one deals with relativistic propagators

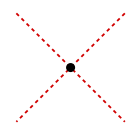

(a)

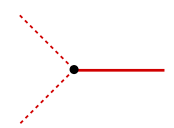

(b)

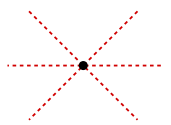

(c)

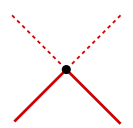

(d)
FIG. 2. Building blocks in the strong sector: (a) LO four-meson contact term, (b) NLO two-meson-resonance coupling, (c) sixmeson contact term, and (d) two-meson-two-resonance coupling.

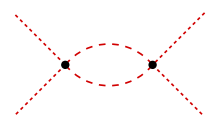

(a)

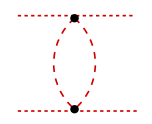

(b)

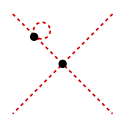

(c)

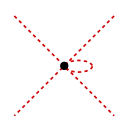

(d)
FIG. 3. One-loop corrections to the contact four-meson vertex: (a) $s$ channel, (b) $t$ and $u$ channels, (c) mass term, and (d) vertex term.

involving mesonic states, denoted by $\Omega$. In order to illustrate this procedure, in Fig. 3 we show some perturbative corrections involving a single loop to the four-meson contact term of diagram (a) in Fig. 2. The diagrams (a) and (b) of Fig. 3 involve propagation between different points, whereas those in diagrams (c) and (d) of Fig. 3 are local and are incorporated into actual values of masses and coupling constants. Our main concern are diagrams (a) and (b) of Fig. 3.

A particularly important point in this constructive approach is that the $s$-channel contribution of Fig. 3(a) is complex and one writes $\Omega(s)=\Omega^{R}(s)+i \Omega^{I}(s)$, where $\Omega^{R}$ and $\Omega^{I}$ are the real and imaginary parts. The function $\Omega^{I}(s)$ is well behaved and underlies imaginary contributions to the FSIs, including resonance widths. In field theory, this kind of imaginary components in some classes of propagators is of fundamental importance, as it is associated with unitarity. A far-reaching consequence is that reliable amplitudes must have a well-defined balance between real and imaginary parts. If this is not the case, they fail to conserve probability, as in some instances of the isobar model. Concerning the real terms $\Omega^{R}$, explicit calculations show that they contain infinite contributions $\Lambda_{\infty}$. Thus, formally, one has $\Omega^{R}=\Omega^{R}+\Lambda_{\infty}$, where $\Omega^{R}$ is a known regular function. The elimination of $\Lambda_{\infty}$ requires renormalization, bringing unknown real constants into the problem. The model presented in this work regards $\Omega^{R}$, the real part of the two-meson propagator.

The study of FSIs in heavy-meson decays relies on nonperturbative amplitudes and their derivation requires the summation of infinite series of perturbative contributions. We exemplify this procedure in the case of a unitary mesonmeson scattering amplitude, denoting the full result by $\mathcal{A}$ and partial contributions with $n$ loops by $\mathcal{A}_{n}$. We begin by defining a kernel $\mathcal{K}_{n}$ as the part of $\mathcal{A}_{n}$ that cannot be separated into two pieces by cutting s-channel two-meson loops only. The first kernel is $\mathcal{K}_{0}$, associated with the tree processes displayed in diagrams (a) of Fig. 4, and it is a real function because at this point we are still dealing with a bare resonance, described by a pole at its mass. The tree amplitude is then given by $\mathcal{A}_{0}=\mathcal{K}_{0}$ and includes crossing symmetry.

The single-loop correction is shown in diagrams (a) and (b) of Fig. 4 and involves three terms in the $s, t$, and $u$ channels. The first one involves a two-meson $s$-channel propagator, whereas the last two do not and are grouped into a new kernel $\mathcal{K}_{1}$. The case of two loops is shown in 
(a)

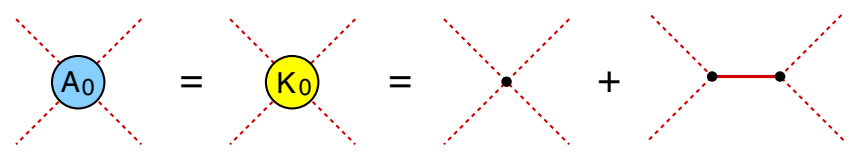

(b)

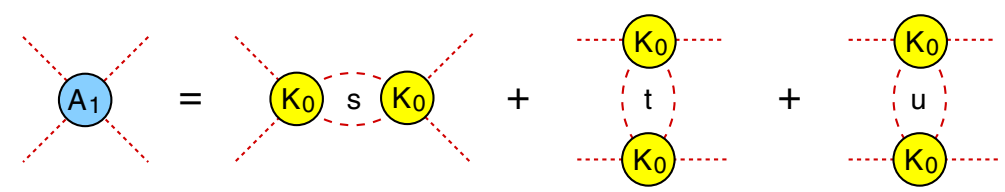

(c)

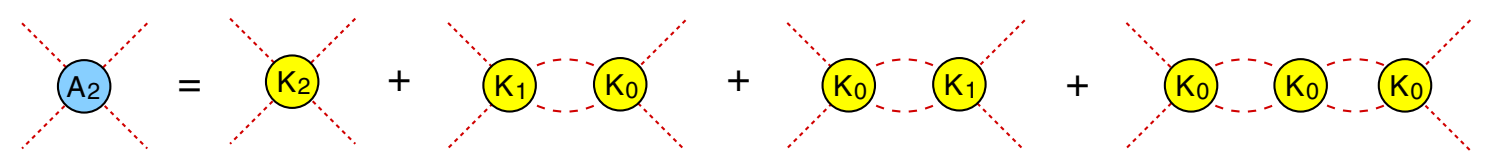

(d)

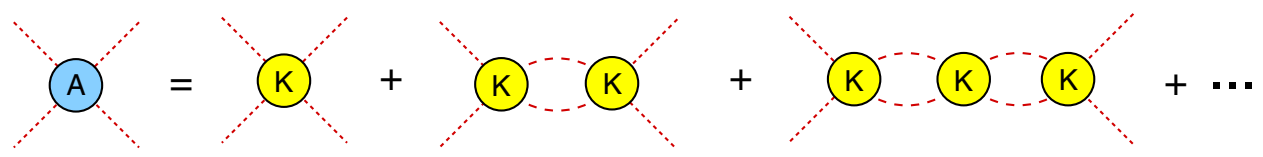

FIG. 4. Scattering amplitudes $\mathcal{A}$ and kernels $\mathcal{K}$ : (a) tree level, (b) first perturbative correction, (c) second perturbative correction, and (d) full amplitude.

diagrams (c) of Fig. 4, where $\mathcal{K}_{2}$ is a higher-order kernel and the $s$-channel is represented by three successive $\mathcal{K}_{0}$ interactions. Repeating this indefinitely and adding the results, we obtain a scattering amplitude of the form

$$
\begin{gathered}
\mathcal{A}=\mathcal{K} \times\left[1+(\text { loop } \times \mathcal{K})+(\text { loop } \times \mathcal{K})^{2}\right. \\
\left.+(\text { loop } \times \mathcal{K})^{3}+\cdots\right], \\
\text { loop }=\Omega^{R}+i \Omega^{I}, \\
\mathcal{K}=\mathcal{K}_{0}+\mathcal{K}_{1}+\mathcal{K}_{2}+\cdots .
\end{gathered}
$$

The geometric series in Eq. (3) can be summed and one has

$$
\begin{gathered}
\mathcal{A}=\frac{\mathcal{K}}{D}, \\
D=1-(\operatorname{loop} \times \mathcal{K}) .
\end{gathered}
$$

As discussed in the sequence, $1 / D$ is the post-QCD version of the BW line shape (2). In practice, the evaluation of the series given by Eq. (5) is unfeasible and, in the framework of chiral perturbation theory, even the structure of the nextto-leading-order (NLO) term $\mathcal{K}_{1}$ is already cumbersome.
So, we keep just the dominant term and rely on the approximation $\mathcal{K} \simeq \mathcal{K}_{0}$.

A very important feature of this result is that the amplitude $\mathcal{A}$ is unitary, provided $\mathcal{K}$ is real. This property is quite general and derives from the structure of the denominator $D$, which is suitably complex owing to the well-defined imaginary function $\Omega^{I}$ in Eq. (4). The forms adopted for both $\Omega^{R}$ and $\mathcal{K}$ are irrelevant for this property of $\mathcal{A}$, as discussed in Sec. IV. This justifies the widespread use of the $K$-matrix approximation, which is implemented by neglecting $\Omega^{R}$ and writing

$$
K \text {-matrix } \rightarrow \text { loop }=0+i \Omega_{I} .
$$

The amplitudes $\mathcal{A}$ are key elements in the description of heavy-meson decays, for they are present in the FSIs which supplement the weak process of Fig. 1. Strong interactions involving three bodies can be very complicated. The simplest class of FSIs corresponds to the $(2+1)$ approximation, represented in Fig. 5, in which the first diagram in Fig. 5(a) represents the nonresonant contribution and the other two include particle interactions in the presence of a final meson acting as a spectator. Diagrams (a) in Fig. 5 represents the heavy-meson decay amplitude in the $(2+1)$ 
(a)

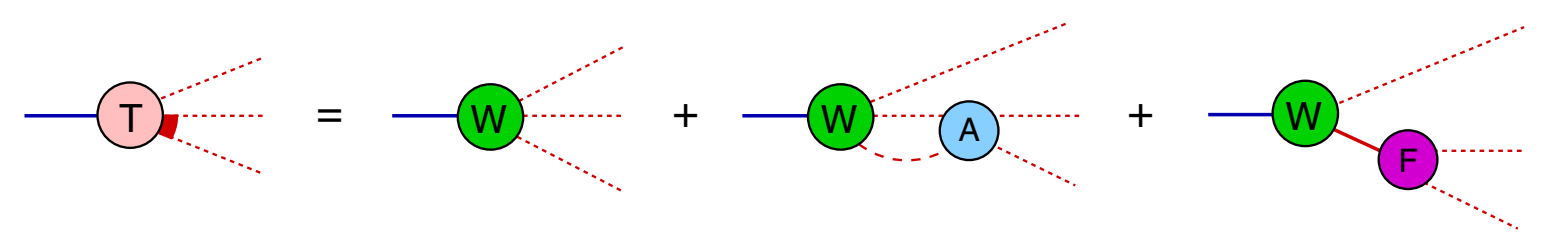

(b)

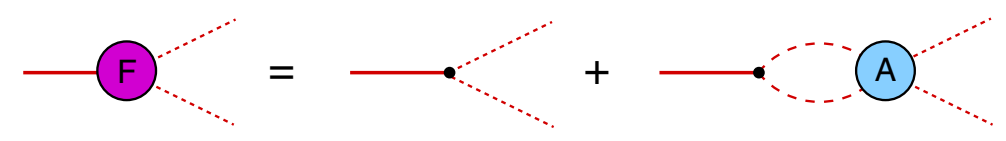

FIG. 5. (a) Decay amplitude in the $2+1$ approximation and (b) the form factor.

approximation and the blob indicated by $F$ is usually called the form factor, which many authors take as the single contribution to the decay [21]. It is isolated in Fig. 5(b) and, denoting by $g$ the resonance-pseudoscalar coupling constant, the function $F$ can be related to the meson-meson scattering amplitude by

$$
F=g[1+(\operatorname{loop} \times A)]=g \frac{1}{D},
$$

where $D$ is the denominator given in Eq. (7). The imaginary part of $D$ gives rise to a finite width to the resonance.

In order to go beyond the $(2+1)$ approximation, one would need to tackle a rather complicated three-body problem, which involves both multiple scattering series and proper three-body interactions, as indicated in Fig. 6. It is worth stressing that these FSIs are not a matter of choice, since they are compulsory contributions to the problem. Part of this sector can be tackled by means of Faddeev techniques [5] or the Khuri-Treiman formalism [7,31] and, in spite of a continuous progress towards a more complete description of three-meson decay dynamics [32], results are still incipient for heavy-meson decays. The Khuri-Treiman formalism, in particular, emphasizes crossing symmetry and can allow one to quantify the implications of the $\mathcal{K} \simeq \mathcal{K}_{0}$ approximation we use, which does not preserve crossing symmetry.
In summary, the decay of a heavy meson into three light mesons involves two distinct sectors: a weak primary vertex and a structure of final-state strong interactions. Although the former is not simple, the latter may be expected to be much more complicated and progress in this area depends on the definition of a hierarchy among strong problems. The simplest subset of problems is provided by the $(2+1)$ approximation and depends on meson-meson scattering amplitudes. Nowadays, even these two-body interactions are not sufficiently well known for systems involving pions, kaons, and etas within the phase space provided by $D$ and $B$ decays.

\section{SCATTERING AMPLITUDES}

In this work we present a practical model for the inclusion of any number of resonances in phenomenological mesonmeson scattering amplitudes, so that they can be used as trial functions in more complicated reactions, such as heavymeson or $\tau$ decays. Instead of presenting the model in its full complexity at once, we choose to construct it gradually, so as to emphasize possible points of contact with the isobar model and point out limitations of the latter.

The scattering amplitudes $A_{(k \ell \mid a b)}^{(J, I)}$ for the process $P_{k} P_{\ell} \rightarrow P_{a} P_{b}$ in a channel with spin $J$ and isospin $I$ are given in Appendix $\mathrm{C}$ and involve four kinds of ingredients:

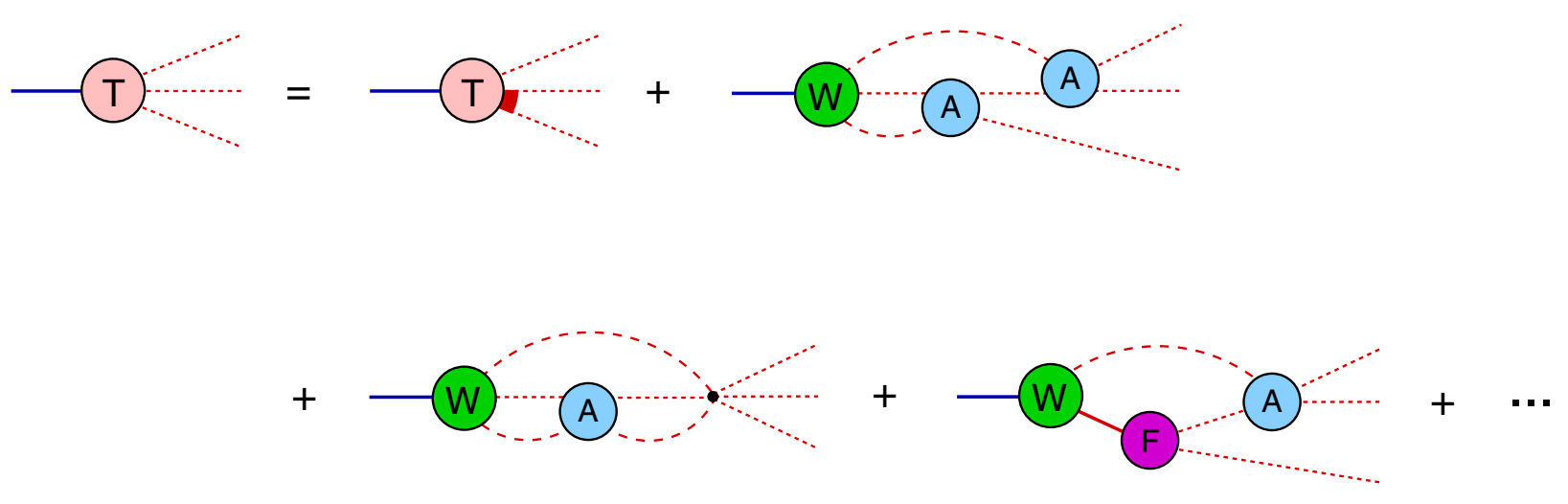

FIG. 6. Decay amplitude: $2+1$ approximation, supplemented by three-body interactions. 
(1) Coupled channels: This sector of the problem is rather standard and model independent. In our notation, the coupling among the various channels is implemented by the mixing matrices $M_{a b}^{(J, I)}$ given by Eqs. (C1)-(C6).

(2) Multiresonance dynamics: The dynamical content of meson-meson $(P P)$ interactions is incorporated into the kernels $\mathcal{K}_{(k l \mid a b)}^{(J, I)}$ given in Appendix B, which are real functions of masses and coupling constants. They include crossed amplitudes at tree level, but no loops in the $t$ and $u$ channels and in the language of Fig. 4 correspond to the approximation $\mathcal{K} \simeq \mathcal{K}_{0}$. While in kernels, resonances have no widths and are characterized just by their poles. The inclusion of several resonances is performed by adding these poles and the reader may want to inspect Eqs. (B31)-(B36) for an example.

(3) Unitarization: We neglect four-meson intermediate states and the unitarization of amplitudes is directly associated with the $s$-channel two-meson propagators $\Omega$ that occur in the full scattering amplitude. These functions, described in Appendix A, contain real and imaginary parts: $\Omega=\Omega^{R}+i \Omega^{I}$. The latter, given by Eqs. (A14)-(A15), are free from ambiguities and constitute the only source of imaginary terms in the amplitudes $A_{(k \ell \mid a b)}^{(J, I)}$. In particular, resonance widths are necessarily proportional to $\Omega^{I}$. The real component of $\Omega^{R}$ has infinite components which are replaced by renormalization constants. The form of this component in the case of several resonances is the object of this work.

(4) Free parameters: The parameters entering our amplitudes consist basically of masses and coupling constants and, in principle, are completely free. Thus, our amplitudes are guess functions with open parameters, to be determined by fits do data. Most of the symbols used to label these parameters were borrowed from chiral perturbation theory, especially Ref. [27]. Their numerical meanings, however, are not exactly the same. In chiral perturbation theory, the values of parameters are extracted by comparing results from calculations performed to a given order with observables. As loops are divergent and need to be renormalized, values for parameters quoted in the literature also depend on renormalization scales. This kind of procedure is theoretically consistent and yields a precise description of low-energy phenomena. In the case of heavy-meson decays, this level of precision cannot be reached because a wider range of energies is involved and perturbation does not apply. Thus, in decay analyses, free parameters do not have the same meaning as their low-energy counterparts, since they are designed to be used in a different mathematical structure. In our amplitudes, free parameters aim at describing the physics within the energy ranges defined by Dalitz plots.

At this point it is worth stressing that the model dependence incorporated in the amplitudes $A_{(k \ell \mid a b)}^{(J, I)}$ given in Appendix $C$ is restricted to the kernels $\mathcal{K}$, which depend on dynamical assumptions, and to the real part $\Omega^{R}$ of twomeson propagators to be discussed in Sec. VI. As the imaginary part $\Omega^{I}$ is unambiguous, the scattering amplitudes are unitary and comply exactly with coupled-channel requirements for any choices made for $\mathcal{K}$ and $\Omega^{R}$. In this sense, the approach tames model dependence as much as possible.

In order to make the discussion more concrete, we concentrate on the case of $\pi \pi$ scattering, described by the amplitudes $A_{(\pi \pi \mid \pi \pi)}^{(J, I)}$, with $(J, I=1,1)$ and $(J, I=0,0)$, for comparisons with the isobar model and discussion of the main features of our model. The extension to other channels is straightforward. Using Eqs. (C12) and (C21), we have

$A_{(\pi \pi \mid \pi \pi)}^{(1,1)}=\frac{(t-u)}{D^{(1,1)}}\left\{\left[1-M_{22}^{(1,1)}\right] \mathcal{K}_{(\pi \pi \mid \pi \pi)}^{(1,1)}+M_{12}^{(1,1)} \mathcal{K}_{(K K \mid \pi \pi)}^{(1,1)}\right\}$,

$$
\begin{aligned}
A_{(\pi \pi \mid \pi \pi)}^{(0,0)}= & \frac{1}{D^{(0,0)}}\left\{\left[\left(1-M_{22}^{(0,0)}\right)\left(1-M_{33}^{(0,0)}\right)\right.\right. \\
& \left.-M_{23}^{(0,0)} M_{32}^{(0,0)}\right] \mathcal{K}_{(\pi \pi \mid \pi \pi)}^{(0,0)} \\
& +\left[M_{12}^{(0,0)}\left(1-M_{33}^{(0,0)}\right)+M_{13}^{(0,0)} M_{32}^{(0,0)}\right] \mathcal{K}_{(K K \mid \pi \pi)}^{(0,0)} \\
& \left.+\left[M_{13}^{(0,0)}\left(1-M_{22}^{(0,0)}\right)+M_{12}^{(0,0)} M_{23}^{(0,0)}\right] \mathcal{K}_{(88 \mid \pi \pi)}^{(0,0)}\right\},
\end{aligned}
$$

where the $\eta$ is represented by 8 . In these results, the complex mixing matrices, given by Eqs. (C1) and (C6), have the general structure $M=\mathcal{K} \times \Omega$. The denominators $D$ contain the pole structure of the theory and have the form

$$
\begin{aligned}
D^{(1,1)}= & {\left[1-M_{11}^{(1,1)}\right]\left[1-M_{22}^{(1,1)}\right]-M_{12}^{(1,1)} M_{21}^{(1,1)} } \\
D^{(0,0)}= & {\left[1-M_{11}^{(0,0)}\right]\left[1-M_{22}^{(0,0)}\right]\left[1-M_{33}^{(0,0)}\right] } \\
& -\left[1-M_{11}^{(0,0)}\right] M_{23}^{(0,0)} M_{32}^{(0,0)} \\
& -\left[1-M_{22}^{(0,0)}\right] M_{13}^{(0,0)} M_{31}^{(0,0)}-\left[1-M_{33}^{(0,0)}\right] M_{12}^{(0,0)} M_{21}^{(0,0)} \\
& -M_{12}^{(0,0)} M_{23}^{(0,0)} M_{31}^{(0,0)}-M_{21}^{(0,0)} M_{13}^{(0,0)} M_{32}^{(0,0)}
\end{aligned}
$$

At low energies, $M_{a b}^{(J, I)} \rightarrow 0$ and the amplitudes (10) and (11) become the real functions 


$$
\begin{aligned}
& A_{(\pi \pi \mid \pi \pi)}^{(1,1)} \rightarrow(t-u) \mathcal{K}_{(\pi \pi \mid \pi \pi)}^{(1,1)} \rightarrow \frac{(t-u)}{F^{2}}, \\
& A_{(\pi \pi \mid \pi \pi)}^{(0,0)} \rightarrow \mathcal{K}_{(\pi \pi \mid \pi \pi)}^{(0,0)} \rightarrow \frac{\left(2 s-M_{\pi}^{2}\right)}{F^{2}},
\end{aligned}
$$

where $F$ is the pion decay constant.

As stressed above, these amplitudes are cast in terms of free parameters, to be obtained from fits to data. Here, in order to explore their features and, in the want of such fitted values, we rely on parameters given in Ref. [27].

\section{STANDARD ISOBAR MODEL: UNCOUPLED CHANNELS}

The form of Eqs. (10) and (11) is involved owing to channel coupling. In order to discuss their contact with the standard isobar model, in this section we pretend that the $\pi \pi$ state cannot couple to $K \bar{K}$ and $\eta \eta$. Labeling the corresponding uncoupled amplitudes with $U$, we have

$$
\begin{aligned}
& A_{(\pi \pi \mid \pi \pi)}^{U(1,1)}=\frac{(t-u) \mathcal{K}_{(\pi \pi \mid \pi \pi)}^{(1,1)}}{1+\mathcal{K}_{(\pi \pi \mid \pi \pi)}^{(1,1)}\left[\Omega_{\pi \pi}^{P} / 2\right]}, \\
& A_{(\pi \pi \mid \pi \pi)}^{U(0,0)}=\frac{\mathcal{K}_{(\pi \pi \mid \pi \pi)}^{(0,0)}}{1+\mathcal{K}_{(\pi \pi \mid \pi \pi)}^{(0,0)}\left[\Omega_{\pi \pi}^{S} / 2\right]},
\end{aligned}
$$

where $\Omega$ are the two-pion propagators discussed in Appendix A. The kernels are given by Eqs. (B1) and (B31) and, in order to simplify the discussion, we assume the value $\epsilon=0$ for the mixing parameter in Eqs. (B29) and (B30). Thus,

$$
\begin{array}{r}
\mathcal{K}_{(\pi \pi \mid \pi \pi)}^{(1,1)}=\frac{1}{F^{2}}-\left[\frac{2 G_{V}^{2}}{F^{4}}\right] \frac{s}{s-m_{\rho}^{2}}-\frac{s G_{\left(\rho^{\prime} \mid \pi \pi\right)}^{2}}{s-m_{\rho^{\prime}}^{2}}, \\
\mathcal{K}_{(\pi \pi \mid \pi \pi)}^{(0,0)}=\frac{\left(2 s-M_{\pi}^{2}\right)}{F^{2}}-\left[\frac{12}{F^{4}}\right] \frac{\left[\tilde{c}_{d} s-\left(\tilde{c}_{d}-\tilde{c}_{m}\right) 2 M_{\pi}^{2}\right]^{2}}{s-m_{S 1}^{2}} \\
-\left[\frac{2}{F^{4}}\right] \frac{\left[c_{d} s-\left(c_{d}-c_{m}\right) 2 M_{\pi}^{2}\right]^{2}}{s-m_{S o}^{2}}-\frac{G_{\left(f^{\prime} \mid \pi \pi\right)}^{2}}{s-m_{f^{\prime}}^{2}}
\end{array}
$$

where $G_{V}, \tilde{c}_{d}, \tilde{c}_{m}, c_{d}, c_{m}$ are coupling constants [27] and $m_{S o}$ and $m_{S 1}$ are the $S U(3)$ octet and singlet scalar resonances. We further simplify these results by considering just a single resonance in each channel. In the vector case, using the approximate identity $G_{V}=F / \sqrt{2}$, one recovers the classic vector meson dominance result [27]

$$
\mathcal{K}_{(\pi \pi \mid \pi \pi)}^{(1,1)} \rightarrow-\frac{m_{\rho}^{2} / F^{2}}{s-m_{\rho}^{2}}
$$

whereas for the scalar one writes

$$
\begin{gathered}
\mathcal{K}_{(\pi \pi \mid \pi \pi)}^{(0,0)} \rightarrow-\frac{\Theta^{2}(s)}{s-m_{S o}^{2}}, \\
\Theta^{2}(s)=\left[-\frac{\left[2 s-M_{\pi}^{2}\right]\left[s-m_{S o}^{2}\right]}{F^{2}}+\frac{2\left[c_{d} s-\left(c_{d}-c_{m}\right) 2 M_{\pi}^{2}\right]^{2}}{F^{4}}\right] .
\end{gathered}
$$

Using Eqs. (20)-(22) with Eqs. (16)-(17) and recalling that the imaginary parts of $\Omega_{\pi \pi}$ are given by Eqs. (A14) and (A15), the uncoupled amplitudes can be expressed in terms of functions $\mathcal{M}$ and $\Gamma$ that resemble masses and widths as

$$
\begin{aligned}
A_{(\pi \pi \mid \pi \pi)}^{U(1,1)} & =-\frac{(t-u) m_{\rho}^{2} / F^{2}}{s-\mathcal{M}_{V}^{2}+i \mathcal{M}_{V} \Gamma_{V}}, \\
\mathcal{M}_{V}^{2} & =m_{\rho}^{2}-\frac{m_{\rho}^{2} \Omega_{\pi \pi}^{P R}}{2 F^{2}}, \\
\mathcal{M}_{V} \Gamma_{V} & =\frac{m_{\rho}^{2}}{96 \pi F^{2}} \frac{\left(s-4 M_{\pi}^{2}\right)^{3 / 2}}{s^{1 / 2}}, \\
A_{(\pi \pi \mid \pi \pi)}^{U(0,0)} & =-\frac{\Theta^{2}(s)}{s-\mathcal{M}_{S}^{2}+i \mathcal{M}_{S} \Gamma_{S}}, \\
\mathcal{M}_{S}^{2} & =m_{S o}^{2}-\frac{\Theta^{2}(s) \Omega_{\pi \pi}^{S R}}{2}, \\
\mathcal{M}_{S} \Gamma_{S} & =\frac{\Theta^{2}(s)}{32 \pi} \frac{\left(s-4 M_{\pi}^{2}\right)^{1 / 2}}{s^{1 / 2}} .
\end{aligned}
$$

These results illustrate a number of features from constructive descriptions of resonances:

(1) Even if we begin with a bare resonance, it acquires a dynamical width by means of interactions with pseudoscalars, whereas the $s$-channel pole present in the kernel becomes complex. In the case of the $\rho$, Eq. (25) yields $\Gamma_{P} \rightarrow \Gamma_{\rho} \sim 145 \mathrm{MeV}$, close to the Particle Data Group value [33].

(2) The functions $\mathcal{M}$ shift the resonance masses from their nominal $m$ values. As indicated by Eqs. (24) and (27), these are model-dependent effects because the real parts $\Omega_{\pi \pi}^{J R}$ of the two-pion propagators contain undetermined free constants, which are remnants of renormalization. A popular way to avoid this problem consists in using the $K$-matrix approach, in which this function is set to zero by fiat. We present our alternative In Sec. VI.

(3) Equations (23) and (26) resemble the Breit-Wigner line shapes given by Eq. (2), but only superficially. In fact, they are rather different because the $\mathcal{M}$ and $\Gamma$ are running functions of $s$. The usual BW expressions, on the other hand, employ masses $\mathcal{M}_{V}^{\mathrm{BW}}=$ $m_{\rho}^{2}, \mathcal{M}_{S}^{\mathrm{BW}}=m_{S o}^{2}$ and widths given by 


$$
\begin{aligned}
\Gamma_{V}^{\mathrm{BW}} & =\frac{\left(s-4 M_{\pi}^{2}\right)^{3 / 2}}{96 \pi F^{2}}, \\
\Gamma_{S}^{\mathrm{BW}} & =C_{S}^{\mathrm{BW}} \frac{\left(s-4 M_{\pi}^{2}\right)^{1 / 2}}{32 \pi},
\end{aligned}
$$

where $C_{S}^{\mathrm{BW}}$ is a coupling constant. Comparing these expressions with Eqs. (25) and (28), we learn that the BW line shape is a good approximation for vector resonances but is unsuited for scalar resonances. The fact that Eq. (25) is identical to the classic GounarisSakurai result produced in 1968 [34] indicates that the vector sector has been stable over the last 50 years. However, the scalar sector is different, because our understanding of it changed significantly after the development of QCD. The ground state of the theory-its vacuum-is not empty and chiral perturbation theory introduces this feature into low-energy physics. In the present case, it gives rise to the incorporation of both contact interactions and $s$-dependent couplings of scalar resonances to pseudoscalars [27] into the function $\Theta(s)$. In this exercise, even if we assume $C_{S}^{\mathrm{BW}}=\Theta^{2}\left(m_{S o}^{2}\right) / m_{S o}^{2}$, the BW approximation for scalars remains unsuited, for all of the rich $s$ dependence of Eq. (22) is lost.

A very important feature of Eqs. (16) and (17) is that they are automatically unitary, irrespective of the features of the kernel $\mathcal{K}$ employed, provided it is real, and of the real part of the two-pion propagator $\Omega_{\pi \pi}^{J R}$. In practice, an easy way to check unitarity is to evaluate the inelasticity $\eta$, using the nonrelativistic amplitudes $f_{(\pi \pi \mid \pi \pi)}^{(J, I)}$ given in Appendix D. Skipping labels, they are related to the $A_{(\pi \pi \mid \pi \pi)}^{(J, I)}$ by

$$
f=-\frac{\Omega^{I}}{2} A_{(\pi \pi \mid \pi \pi)}^{(J, I)},
$$

where the $\Omega^{I}$ are the imaginary parts of the two-pion propagator, given by Eqs. (A14) and (A15). Thus, one has the generic form

$$
\begin{gathered}
f=-\frac{1}{w+i}, \\
w=\frac{1+\mathcal{K} \Omega^{R} / 2}{\mathcal{K} \Omega^{I} / 2} .
\end{gathered}
$$

Unitarity is ensured because, for any function of the form (32), irrespective of the value of $w$, the inelasticity parameter [given by Eq. (D8)] is always $\eta=1$ in the absence of other channels. So, this is a model-independent result, valid for any choices of $\mathcal{K}$ and $\Omega^{R}$.

One now considers the case of several resonances in the same channel. As shown in Appendix B, the kernel for a channel containing $n$ resonances represented by individual terms $\mathcal{K}_{j}$ is written as

$$
\mathcal{K}=\mathcal{K}_{c}+\mathcal{K}_{1}+\cdots+\mathcal{K}_{n}
$$

where $\mathcal{K}_{c}$ is a contact term. Using Eqs. (16), (17), and (31), we write the nonrelativistic amplitude as

$$
\begin{gathered}
f=-\frac{\left[\mathcal{K}_{c}+\mathcal{K}_{1}+\cdots+\mathcal{K}_{n}\right] \Omega^{I} / 2}{1+\left[\mathcal{K}_{c}+\mathcal{K}_{1}+\cdots+\mathcal{K}_{n}\right]\left[\Omega^{R}+i \Omega^{I}\right] / 2} \\
=-\frac{1}{w+i}, \\
w=\frac{1+\left[\mathcal{K}_{c}+\mathcal{K}_{1}+\cdots+\mathcal{K}_{n}\right] \Omega^{R} / 2}{\left[\mathcal{K}_{c}+\mathcal{K}_{1}+\cdots+\mathcal{K}_{n}\right] \Omega^{I} / 2} .
\end{gathered}
$$

This amplitude is unitary because this property does not depend on the form of the kernel.

In the standard isobar model, on the other hand, unitarized resonances are treated individually and, for each of them, one would write

$$
\begin{gathered}
f_{i}=-\frac{\mathcal{K}_{i} \Omega^{I} / 2}{1+\mathcal{K}_{i}\left[\Omega^{R}+i \Omega^{I}\right] / 2}=-\frac{1}{w_{j}+i}, \\
w_{j}=\frac{1+\mathcal{K}_{j} \Omega^{R} / 2}{\mathcal{K}_{j} \Omega^{I} / 2} .
\end{gathered}
$$

These unitary terms are then added schematically as $f_{\text {model }}^{\text {isobar }}=\alpha_{c} f_{c}+\alpha_{1} f_{1}+\cdots+\alpha_{n} f_{n}$, where the $\alpha$ are complex functions of $s$. Thus, one has $f \neq f_{\text {model }}^{\text {isobar }}$ and learns that the standard isobar model prescription for adding resonances is not compatible with unitarity. This happens because it treats each resonance as an individual object, whereas in the amplitude they are necessarily coupled among themselves by the intermediate states that they share. Unitarity is a global property that cannot be split as sums of individual contributions.

In summary, the addition of resonances and unitarization does not commute and, after QCD, the SIM structure is suited just for the case of a single uncoupled vector resonance.

\section{RESONANCES: COUPLED CHANNELS}

The qualitative features of coupled channels are discussed just in the case of the scalar-isoscalar amplitude $A_{(\pi \pi \mid \pi \pi)}^{(0,0)}$, including $K K$ and $\eta \eta$ couplings, given by Eq. (11) and cast in the form

$$
\begin{gathered}
A_{(\pi \pi \mid \pi \pi)}^{(0,0)}=\frac{N_{(\pi \pi \mid \pi \pi)}^{(0,0)}}{D^{(0,0)}}, \\
N_{(\pi \pi \mid \pi \pi)}^{(0,0)}=\mathcal{K}_{(\pi \pi \mid \pi \pi)}^{(0,0)}+C_{(\pi \pi \mid K K)}^{(0,0)} \Omega_{K K}^{S} / 2 \\
+C_{(\pi \pi \mid 88)} \Omega_{88}^{S} / 2+C_{(\pi \pi|K K| 88)} \Omega_{K K}^{S} \Omega_{88}^{S} / 4,
\end{gathered}
$$




$$
\begin{aligned}
D^{(0,0)}= & 1+\left[\mathcal{K}_{(\pi \pi \mid \pi \pi)}^{(0,0)} \Omega_{\pi \pi}^{S} / 2+\mathcal{K}_{(K K \mid K K)}^{(0,0)} \Omega_{K K}^{S} / 2\right. \\
+ & \left.\mathcal{K}_{(88 \mid 88)}^{(0,0)} \Omega_{88}^{S} / 2\right]+C_{(\pi \pi \mid K K)} \Omega_{\pi \pi}^{S} \Omega_{K K}^{S} / 4 \\
+ & C_{(\pi \pi \mid 88)} \Omega_{\pi \pi}^{S} \Omega_{88}^{S} / 4+C_{(K K \mid 88)} \Omega_{K K}^{S} \Omega_{88}^{S} / 4 \\
+ & C_{(\pi \pi|K K| 88)} \Omega_{\pi \pi}^{S} \Omega_{K K}^{S} \Omega_{88}^{S} / 8 \\
C_{(\pi \pi \mid K K)}= & \mathcal{K}_{(\pi \pi \mid \pi \pi)}^{(0,0)} \mathcal{K}_{(K K \mid K K)}^{(0,0)}-\left[\mathcal{K}_{(\pi \pi \mid K K)}^{(0,0)}\right]^{2}, \\
C_{(\pi \pi \mid 88)}= & \mathcal{K}_{(\pi \pi \mid \pi \pi)}^{(0,0)} \mathcal{K}_{(88 \mid 88)}^{(0,0)}-\left[\mathcal{K}_{(\pi \pi \mid 88)}^{(0,0)}\right]^{2}, \\
C_{(K K \mid 88)}= & \mathcal{K}_{(K K \mid K K)}^{(0,0)} \mathcal{K}_{(88 \mid 88)}^{(0,0)}-\left[\mathcal{K}_{(K K \mid 88)}^{(0,0)}\right]^{2}, \\
C_{(\pi \pi|K K| 88)}= & \mathcal{K}_{(\pi \pi \mid \pi \pi)}^{(0,0)} \mathcal{K}_{(K K \mid K K)}^{(0,0)} \mathcal{K}_{(88 \mid 88)}^{(0,0)}-\mathcal{K}_{(\pi \pi \mid \pi \pi)}^{(0,0)}\left[\mathcal{K}_{(K K \mid 88)}^{(0,0)}\right]^{2} \\
& \quad-\mathcal{K}_{(K K \mid K K)}^{(0,0)}\left[\mathcal{K}_{(\pi \pi \mid 88)}^{(0,0)}\right]^{2}-\mathcal{K}_{(88 \mid 88)}^{(0,0)}\left[\mathcal{K}_{(\pi \pi \mid K K)}^{(0,0)}\right]^{2} \\
& +2 \mathcal{K}_{(\pi \pi \mid K K)}^{(0,0)} \mathcal{K}_{(\pi \pi \mid 88)}^{(0,0)} \mathcal{K}_{(K K \mid 88)}^{(0,0)} .
\end{aligned}
$$

\section{A. Close to the poles}

The kernels $\mathcal{K}_{(a a \mid b b)}^{(0,0)}$ involving three bare poles are displayed in Appendix $\mathrm{B}$ and a naive inspection of Eqs. (40)-(45) could suggest that the amplitude (39) would be highly singular. However, this is not the case. In order to simplify the discussion, we assume that the mixing angle $\epsilon=0$ in Eqs. (B29) and (B30) and, at the vicinity of a pole (be it $S_{o}, S_{1}$, or $S^{\prime}$ ) the kernels have the general structure

$$
\mathcal{K}_{(a a \mid b b)}^{(0,0)} \simeq-\frac{G_{a a} G_{b b}}{\Delta}-B_{a a b b},
$$

$$
\Delta=\left(s-m^{2}\right),
$$

where the $B_{a a b b}$ are finite backgrounds and redundant indices are skipped. Below, we show that divergent terms proportional to $\Delta^{-2}$ and $\Delta^{-3}$ cancel out in both $N_{(\pi \pi \mid \pi \pi)}^{(0,0)}$ and $D^{(0,0)}$ and the amplitude $A_{(\pi \pi \mid \pi \pi)}^{(0,0)}$ is finite at the pole. Close to the pole, explicit calculation yields

$$
\begin{aligned}
N_{(\pi \pi \mid \pi \pi)}^{(0,0)} \simeq & \frac{1}{\Delta}\left\{-\left[G_{\pi \pi}^{2}+\Delta B_{\pi \pi \pi \pi}\right]\right. \\
& +H_{(\pi \pi \mid K K)} \Omega_{K K}^{S} / 2+H_{(\pi \pi \mid 88)} \Omega_{88}^{S} / 2 \\
& \left.\quad-H_{(\pi \pi|K K| 88)} \Omega_{K K}^{S} \Omega_{88}^{S} / 4+\Delta[\cdots]\right\} \\
D^{(0,0)} \simeq & \frac{1}{\Delta}\left\{\left(s-m^{2}\right)-\left(G_{\pi \pi}^{2}+\Delta B_{\pi \pi \pi \pi}\right) \Omega_{\pi \pi}^{S} / 2\right. \\
- & \left(G_{K K}^{2}+\Delta B_{K K K K}\right) \Omega_{K K}^{S} / 2-\left(G_{88}^{2}+\Delta B_{8888}\right) \Omega_{88}^{S} / 2 \\
+ & H_{(\pi \pi \mid K K)} \Omega_{\pi \pi}^{S} \Omega_{K K}^{S} / 4+H_{(\pi \pi \mid 88)} \Omega_{\pi \pi}^{S} \Omega_{88}^{S} / 4 \\
+ & H_{(K K \mid 88)} \Omega_{K K}^{S} \Omega_{88}^{S} / 4 \\
- & \left.H_{(\pi \pi|K K| 88)} \Omega_{\pi \pi}^{S} \Omega_{K K}^{S} \Omega_{88}^{S} / 8+\Delta[\cdots]\right\} \\
H_{(\pi \pi \mid K K)}= & G_{\pi \pi}^{2} B_{K K K K}+G_{K K}^{2} B_{\pi \pi \pi \pi}-2 G_{\pi \pi} G_{K K} B_{\pi \pi K K}, \\
H_{(\pi \pi \mid 88)}= & G_{\pi \pi}^{2} B_{8888}+G_{88}^{2} B_{\pi \pi \pi \pi}-2 G_{\pi \pi} G_{88} B_{\pi \pi 88} \\
H_{(K K \mid 88)}= & G_{K K}^{2} B_{8888}+G_{88}^{2} B_{K K K K}-2 G_{K K} G_{88} B_{K K 88},
\end{aligned}
$$

These results show that, at the pole, both $N_{(\pi \pi \mid \pi \pi)}^{(0,0)}$ and $D^{(0,0)}$ diverge as $1 / \Delta$ and yield a finite amplitude, as expected. They also shed light on a conceptual limitation of the isobar model. Since the functions $H$ involve products of coupling constants $G$ and background contributions $B$ from other channels, resonances no longer behave as individual objects. This contradicts the tacit assumption underlying the isobar model, namely, that background terms can be neglected and resonances can be isolated.

In order to check the importance of background terms, we consider the case of a hypothetical single octet resonance of mass $m=1.05 \mathrm{GeV}$, between the $K K$ and 88 thresholds, where the finite backgrounds are given just by the chiral LO contact terms in Eqs. (B31)-(B34), with opposite signs. Using the coupling constants prescribed in Ref. [27], the nonvanishing contributions come from $G_{\pi \pi}=8.06 \mathrm{GeV}, G_{K K}=10.76 \mathrm{GeV}, B_{\pi \pi \pi \pi}=-252.69$, $B_{\pi \pi K K}=-110.39$, and $B_{K K K K}=-191.21$, which yield $G_{\pi \pi}^{2}=64.93 \mathrm{GeV}^{2}, G_{K K}^{2}=115.69 \mathrm{GeV}^{2}$, and $H_{\pi \pi K K}=$ $-22513.61 \mathrm{GeV}^{2}$. We adopt the $K$-matrix approximation, which consists in setting $\Omega^{R}=0$ and keeping only $\Omega^{I}$. Using $\left[\Omega_{\pi \pi}^{S}\right]^{I}=-191.78 \times 10^{-4}$ and $\left[\Omega_{K K}^{S}\right]^{I}=-67.69 \times$ $10^{-4}$, one finds $N_{(\pi \pi \mid \pi \pi)}^{(0,0)}=\{-64.93+[i 76.20]\} / \Delta$ and $D^{(0,0)}=\{i 0.62+i 0.39+[0.73]\} / \Delta$, where the contributions involving the background are indicated by $[\cdots]$. They cannot be neglected, indicating that Breit-Wigner line shapes [Eq. (2)] are not suited for describing resonances above a crossing threshold. 


\section{B. $K$-matrix results}

As already stressed, the imaginary component $\Omega^{I}$ of the two-meson propagators $\Omega$ is fully determined by theory. In the widely used $K$-matrix approach, only this part is kept and the choice $\Omega^{R}=0$ amounts, in fact, to a disguised model for the real part. In the case of uncoupled channels, this choice has the advantage of allowing a clear identification of the nominal value of the resonance mass. In this subsection, we present numerical studies for the scalarisoscalar amplitude $A_{(\pi \pi \mid \pi \pi)}^{(0,0)}$ given by Eq. (11) and rely on expressions for the kernel given in Appendix B, with resonance masses $m_{f a}=1.37 \mathrm{GeV}, m_{f b}=0.98 \mathrm{GeV}$, and coupling parameters fixed in Ref. [27]. Once the value of $\Omega^{R}$ is fixed, predictions only depend on the models used for the interaction kernel.

In Fig. 7 we neglect $K \bar{K}$ and $\eta \eta$ couplings and compare results from two versions of Eq. (B31), both with $\epsilon=0$. One of them keeps just its third term, representing an octet resonance $(\mathrm{R})$, while the other also includes the first term, describing a contact chiral interaction $(\mathrm{C}+\mathrm{R})$, which is one of the signatures of post-QCD physics. In the jargon of the isobar model, the resonant structure corresponds to a BW line shape, as discussed in Sec. IV. One notes that the contact term is rather important and the dominance of the resonance is restricted to a narrow band around its mass $m_{f b}$. Close to threshold, the chiral contribution yields Eq. (15) and gives the correct magnitude for the scattering length.

The opening of the $K \bar{K}$ channel is studied in Fig. 8, for the same $\mathrm{C}+\mathrm{R}$ case considered before, keeping the resonance mass fixed at $m_{f b}=0.98 \mathrm{GeV}$, while adopting two fake values for $M_{K}$, namely, 0.48 and $0.50 \mathrm{GeV}$, so as to have the $K \bar{K}$ threshold both below and above it.

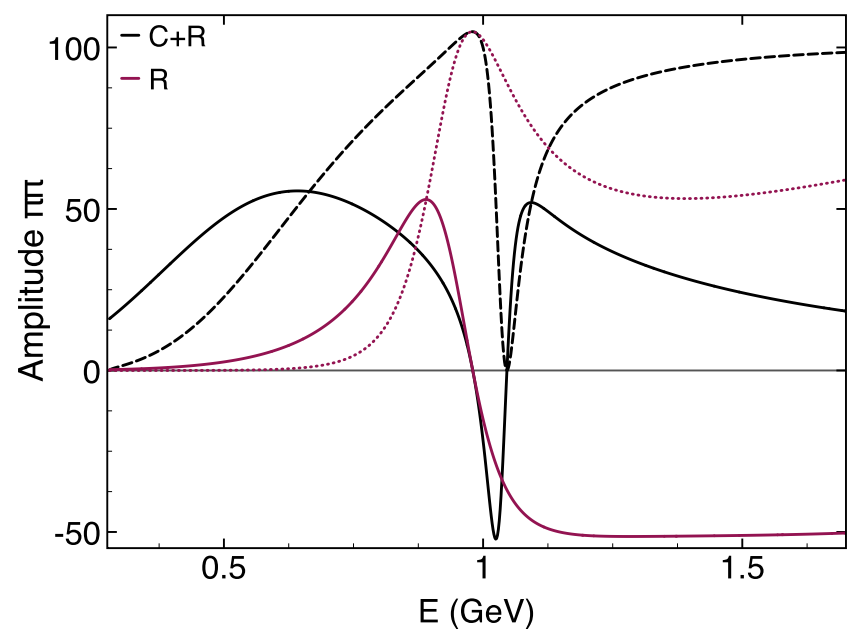

FIG. 7. Predictions for real (full curves) and imaginary (dashed curves) parts of the scalar-isoscalar $\pi \pi$ amplitude based on a single resonance $(\mathrm{R})$ and the same resonance superimposed to a chiral contact term $(C+R)$.

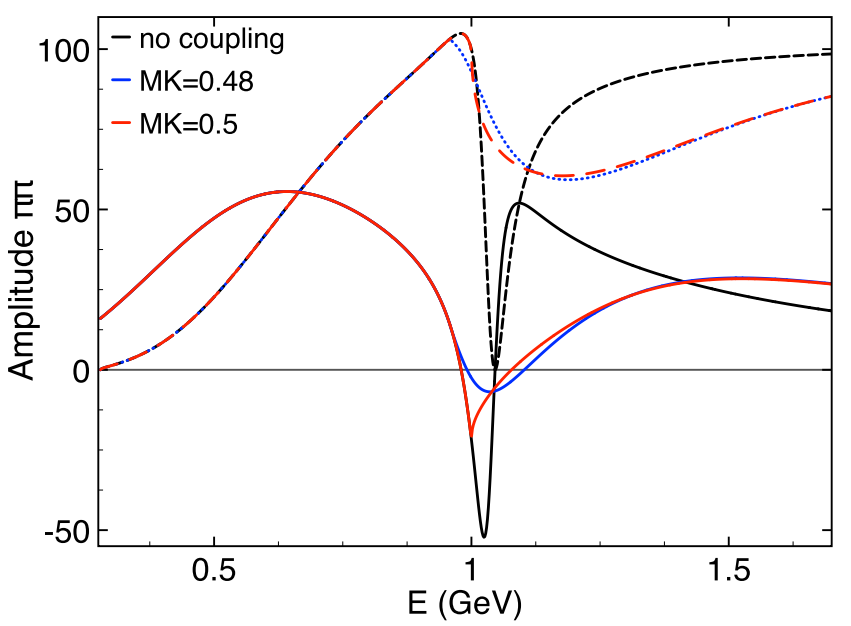

FIG. 8. Predictions for real (full curves) and imaginary (dashed curves) parts of the scalar-isoscalar $\pi \pi$ amplitude based on a single resonance superimposed to a nonresonant background $(\mathrm{NR}+\mathrm{R})$ for no coupled channels (black) and a coupled $K \bar{K}$ channel with threshold below (blue) and above (red) the resonance mass.

As expected, all curves coincide below the thresholds. Above them, however, one learns that the impact of the coupling is important, since the previous $\mathrm{C}+\mathrm{R}$ form provides a very poor representation for the new results, irrespective of the value of $M_{K}$ chosen. At threshold, one has a usual cusp in the real part of the amplitude for $m_{f b}<2 M_{K}$ and a discontinuity in its imaginary part for $m_{f b}>2 M_{K}$. Beyond that point, the real curves display the upward bending associated with the polynomial chiral background, whereas usual connections between real and imaginary parts are lost owing to inelastic effects.

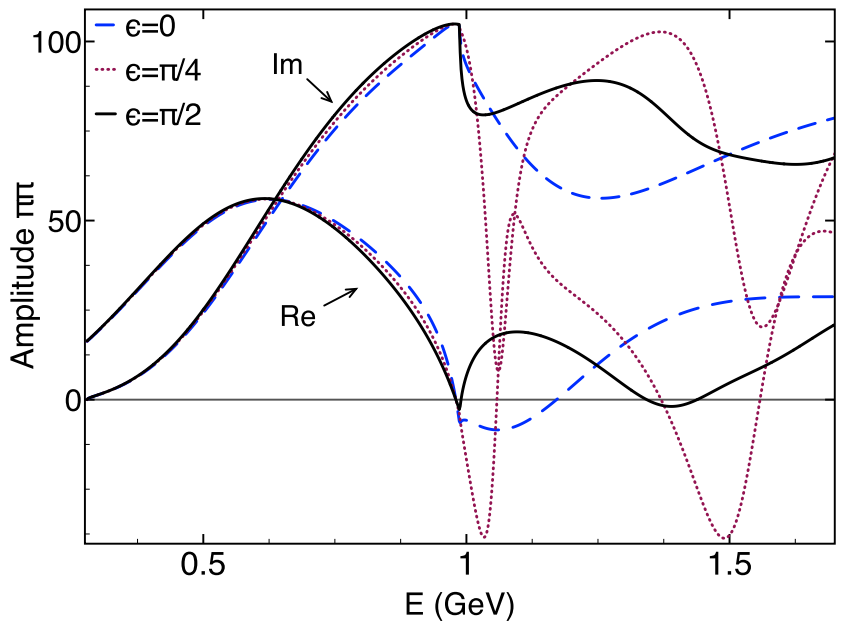

FIG. 9. Predictions for real and imaginary parts of the scalarisoscalar $\pi \pi$ amplitude based on two resonances superimposed to a nonresonant background $\left(\mathrm{NR}+f_{a}+f_{b}\right)$ with a coupled $K \bar{K}$ channel, for mixing parameter $\epsilon=0$ (full lines), $\epsilon=\pi / 4$ (dotted lines), and $\epsilon=\pi$ (dashed lines). 

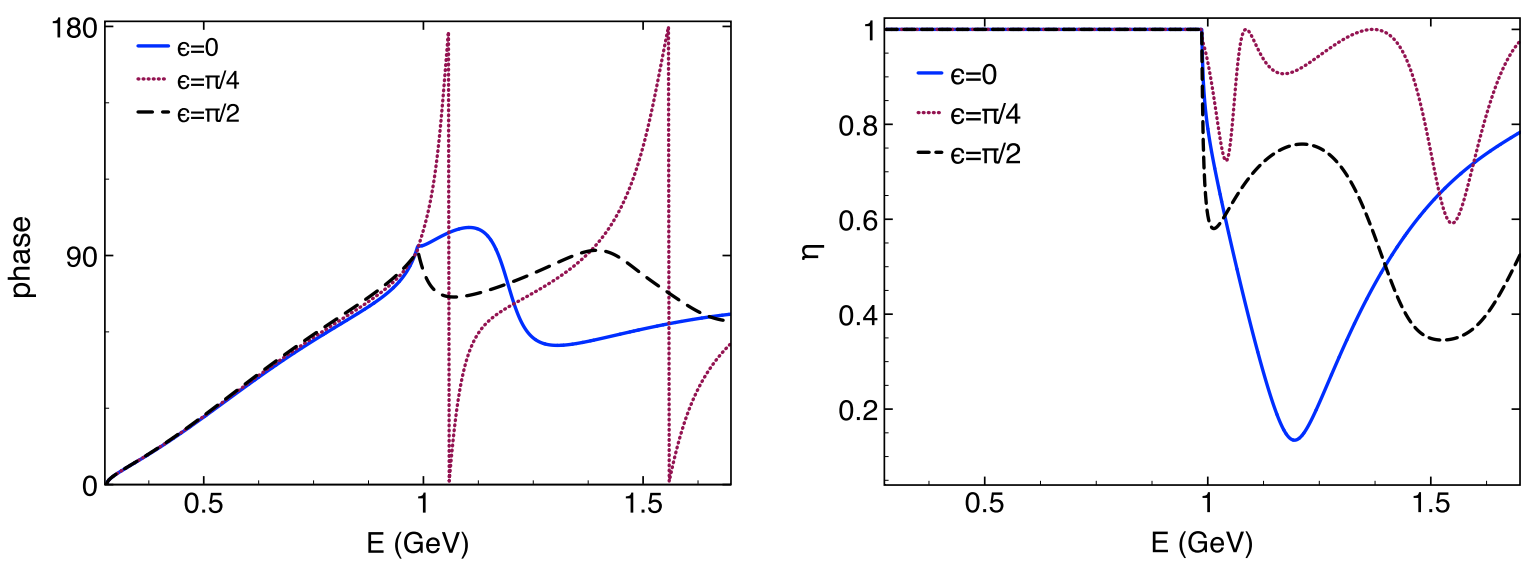

FIG. 10. Predictions for the scalar-isoscalar $\pi \pi$ phase shift $\delta^{(0,0)}$ (left) and inelasticity parameter $\eta^{(0,0)}$ (right) based on two resonances superimposed to a nonresonant background $\left(\mathrm{C}+f_{a}+f_{b}\right)$ with a coupled $K \bar{K}$ channel, for mixing parameters $\epsilon=0$ (full lines), $\epsilon=\pi / 4$ (dotted lines), and $\epsilon=\pi$ (dashed lines).

Altogether, the shift in $M_{K}$ affects the amplitudes only in a narrow region of about $200 \mathrm{MeV}$ above threshold.

In the scalar-isoscalar sector, $S U(3)$ gives rise to octet and singlet states $S o$ and $S 1$, which can be combinations of the observed resonances $f_{a}=f(1370)$ and $f_{b}=f(980)$, with a mixing angle $\epsilon$ defined by Eqs. (B29) and (B30). The influence of this parameter on the $\pi \pi$ amplitude is shown in Fig. 9 for two resonances superimposed to the chiral background, adopting $\epsilon=0, \pi / 4, \pi / 2$. All curves coincide up to $E=0.98 \mathrm{GeV}$, but become quite different afterwards, with the most striking feature being the change in the number of zeros of the real part over the energy range considered. The influence of the mixing angle over the phase shift $\delta^{(0,0)}$ and inelasticity parameter $\eta^{(0,0)}$ is presented in Fig. 10.

\section{MODEL FOR TWO-MESON PROPAGATOR}

The discussion presented here is general and applies to all meson-meson channels. The amplitudes given in Appendix $\mathrm{C}$ are model dependent through both the kernels $\mathcal{K}$ and the real components $\Omega^{R}$ of the two-meson propagators $\Omega=\Omega^{R}+i \Omega^{I}$. The dependence on $\mathcal{K}$ has a dynamical character, since it relies on interactions and parameters from Lagrangians, such as masses and coupling constants, whereas the $\Omega^{R}$ discussed here is a phenomenological model.

The intermediate two-meson propagators for states $a$ and $b$ are given in Appendix A [Eqs. (A11) and (A12)] and their complex forms for $J=0,1$ read

$$
\begin{gathered}
\Omega_{a b}^{S}=-\frac{\Pi_{a b}(s)}{16 \pi^{2}}, \\
\Omega_{a b}^{P}=-\frac{\lambda}{48 \pi^{2} s} \Pi_{a b}(s),
\end{gathered}
$$

where $\lambda$ is the Källén function, and $\Pi_{a b}$ represents the regular parts of loop integrals, which are determined by theory and shown in Eqs. (A4)-(A9). Owing to renormalization, the real parts of the functions $\Omega$ must be supplemented by arbitrary constants to be fixed by experiment, and that is why a model dependence comes in. In the framework of chiral perturbation theory, these constants are coefficients of polynomials on external momenta [16].

The model introduced here consists in a generalization scheme for Eqs. (54) and (55) and its explicit form depends on the number of resonances considered, which are denoted by $R_{x}, R_{y}, R_{z} \cdots$. Their masses and coupling constants are taken as free parameters so that they can be fitted in phenomenological analyses.

In order to motivate the choices we have made, we consider the case $J=0$ and begin with the case of a single resonance, which is written as

$$
\Omega_{a b}^{S}(s) \rightarrow \frac{1}{16 \pi^{2}}\left\{\left[F_{x}(s) \Pi_{a b}^{R}\left(m_{x}^{2}\right)\right]-\Pi_{a b}(s)\right\},
$$

where the term within square brackets is real and corresponds to a subtraction. It generalizes an expression employed earlier in the study of the $K \pi$ amplitude [6]. The function $F_{x}(s)$ is a form factor that satisfies the following conditions.

(a) $F_{x}(s) \rightarrow 0$ for $s \rightarrow 0$ : This is important to ensure that loop corrections do not spoil chiral symmetry results at low energies. In that region, the symmetry predicts amplitudes that are proportional to the real contact terms present in the kernels given in Appendix B, and therefore the functions $\Omega$ cannot show up there.

(b) $F_{x}(s)=1$ for $s=m_{x}^{2}$ : This condition implies that the real component satisfies $\Omega_{a b}^{S R}\left(m_{x}^{2}\right)=0$ and was chosen with practical purposes in mind, so that results coincide with those of the $K$-matrix approach at $s=m_{x}^{2}$. In the case of uncoupled channels, this allows the nominal mass of the resonance to be identified with a zero of the real part of the scattering amplitude. In the case of coupled channels, this property is preserved 

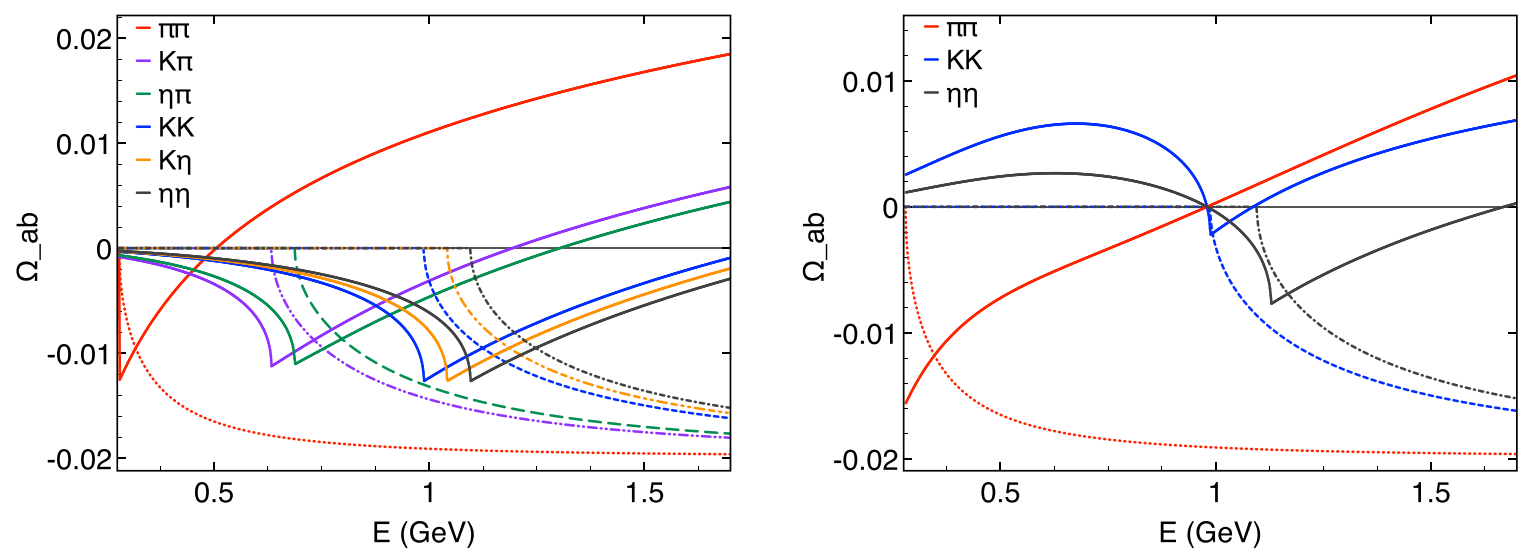

FIG. 11. Behavior of the real (continuous lines) and imaginary (dashed lines) parts of two-meson propagators: (left) functions $\Omega_{\pi \pi}^{S}$, $\Omega_{K \pi}^{S}, \Omega_{\pi 8}^{S}, \Omega_{K K}^{S}, \Omega_{K 8}^{S}$, and $\Omega_{88}^{S}$ from Eq. (54); (right) model predictions for the isospin-0 channel, based on a single $f_{b}$ resonance of mass $m_{f_{b}}=0.98 \mathrm{GeV}$, from Eq. (56).

in the elastic regime below the first threshold but changes afterwards, as shown in Fig. 9. The subtraction performed at the resonance mass is a conservative one, intended to prevent the increase of free parameters in the model.

(c) $F_{x}(s)$ is finite for $s \rightarrow \infty$ : Chiral symmetry holds at low energies only, where it requires subtraction terms as polynomials in $s$. However, these may become too important at high energies where the theory is no longer valid, and this unwanted behavior is avoided by imposing the form factor to be bound in that limit.

The class of functions satisfying these criteria is, of course, very large and our choice is

$$
F_{x}(s)=\frac{4 m_{x}^{2} s}{\left(s+m_{x}^{2}\right)^{2}}
$$

which has a maximum at $s=m_{x}^{2}$. In the left panel of Fig. 11 we show the energy dependence of the two-meson propagators for $\pi \pi, K \pi, \pi \eta, K \bar{K}, K \eta$, and $\eta \eta$ states given by

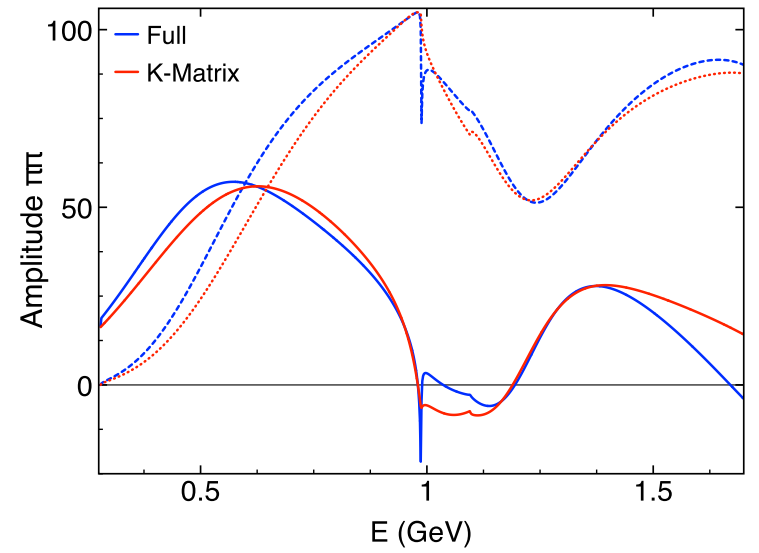

Eq. (54), where it is possible to see the different scales associated with $S U(2)$ and $S U(3)$ sectors. In the right panel we present model predictions based on Eq. (56) for the isospin- 0 channel, based on a single $f_{b}$ resonance of mass $m_{f_{b}}=0.98 \mathrm{GeV}$. We notice that the subtraction makes the real parts of $\Omega^{S}$ vanish at the resonance mass and that the effects of the form factor $F_{x}(s)$ are more important at low energies, the very region where the functions $\Omega$ are less important owing to chiral symmetry. These combined features suggest that the overall influence of the specific choice made in Eq. (57) is expected to be small.

The extensions of Eq. (56) to the case of two and three resonances read

$$
\begin{aligned}
\Omega_{a b}^{S}(s) \rightarrow & \frac{1}{16 \pi^{2}}\left\{F_{x}(s) \frac{\left(s-m_{y}^{2}\right)}{\left(m_{x}^{2}-m_{y}^{2}\right)} \Pi_{a b}^{R}\left(m_{x}^{2}\right)\right. \\
& \left.+F_{y}(s) \frac{\left(m_{x}^{2}-s\right)}{\left(m_{x}^{2}-m_{y}^{2}\right)} \Pi_{a b}^{R}\left(m_{y}^{2}\right)-\Pi_{a b}(s)\right\},
\end{aligned}
$$

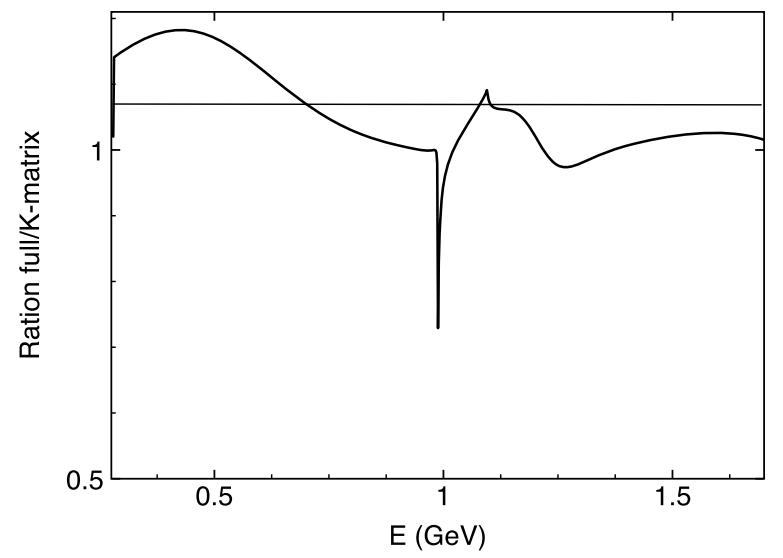

FIG. 12. Predictions for the scalar-isoscalar $\pi \pi$ amplitude with two resonances $f_{a}(1370)$ and $f_{b}(980)$, with $\epsilon=0$, superimposed to a nonresonant background from the model (58) (blue) and the $K$ matrix (red). Left: real (full curve) and imaginary (dashed curve) parts. Right: ratio of magnitudes. 

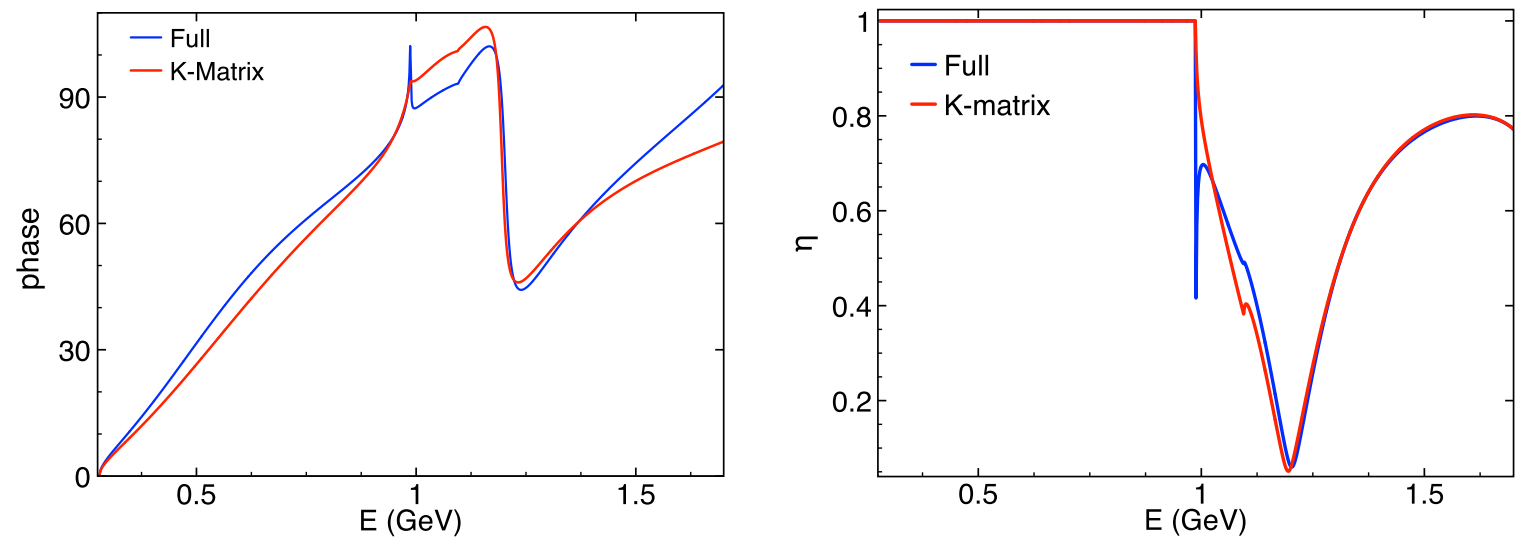

FIG. 13. Predictions for phase shifts (left) and inelasticity parameter (right) of the scalar-isoscalar $\pi \pi$ amplitude with two resonances $f_{a}(1370)$ and $f_{b}(980)$, with $\epsilon=0$, superimposed to a nonresonant background: from the model (58) (blue) and the $K$ matrix (red).

$$
\begin{aligned}
\Omega_{a b}^{S}(s) & \rightarrow \frac{1}{16 \pi^{2}}\left\{F_{x}(s) \frac{\left(s-m_{y}^{2}\right)\left(s-m_{z}^{2}\right)}{\left(m_{x}^{2}-m_{y}^{2}\right)\left(m_{x}^{2}-m_{z}^{2}\right)} \Pi_{a b}^{R}\left(m_{x}^{2}\right)\right. \\
& +F_{y}(s) \frac{\left(m_{x}^{2}-s\right)\left(s-m_{z}^{2}\right)}{\left(m_{x}^{2}-m_{y}^{2}\right)\left(m_{y}^{2}-m_{z}^{2}\right)} \Pi_{a b}^{R}\left(m_{y}^{2}\right) \\
& \left.+F_{z}(s) \frac{\left(m_{x}^{2}-s\right)\left(m_{y}^{2}-s\right)}{\left(m_{x}^{2}-m_{z}^{2}\right)\left(m_{y}^{2}-m_{z}^{2}\right)} \Pi_{a b}^{R}\left(m_{z}^{2}\right)-\Pi_{a b}(s)\right\} .
\end{aligned}
$$

The corresponding expressions for the $J=1$ case $\Omega_{a b}^{P}$ can be obtained from Eqs. (56), (58), and (59) through multiplication by a factor of $\lambda / 3 s$.

We compare predictions from the model and the $K$ matrix for the scalar-isoscalar $\pi \pi$ amplitude in Fig. 12, for the case of two resonances $f_{a}=f(1370)$ and $f_{b}=f(980)$ with the mixing parameter $\epsilon=0$. The corresponding phase shift and inelasticity parameter are shown in Fig. 13. It is possible to notice that results from the model and the $K$ matrix are qualitatively similar over the energy range considered, except for a small region around $1 \mathrm{GeV}$ where effects from the resonance $f_{b}$ and the opening of the $K \bar{K}$ channel compete. This can be seen more clearly in the sharp peak in the figure for the phase, whose tip occurs at threshold. For slightly lower energies, the resonance tends to push the phase upwards, whereas the coupled $K \bar{K}$ interaction does the opposite afterwards. In order to explore this picture, we use a slightly lower mass for the octet resonance, namely, $f_{b}=0.96 \mathrm{GeV}$, and Figs. 14 and 15 show that effects near threshold become much stronger. The phase for the model, in particular, has a sharp rise around $1 \mathrm{GeV}$, as shown in Fig. 15 and also observed by experiment [15], but this does not happen for the $K$ matrix. Another interesting feature of this channel concerns the second resonance $f_{a}(1370)$. Inspecting Figs. 11-14 around the corresponding energy, we do not find structures in either the amplitudes or phase shifts and inelasticities. As both the $K K$ and $\eta \eta$ channels are already open at the $f_{a}$ mass, its pole occurs in the presence of a background due to a chiral contact term superimposed to the resonance
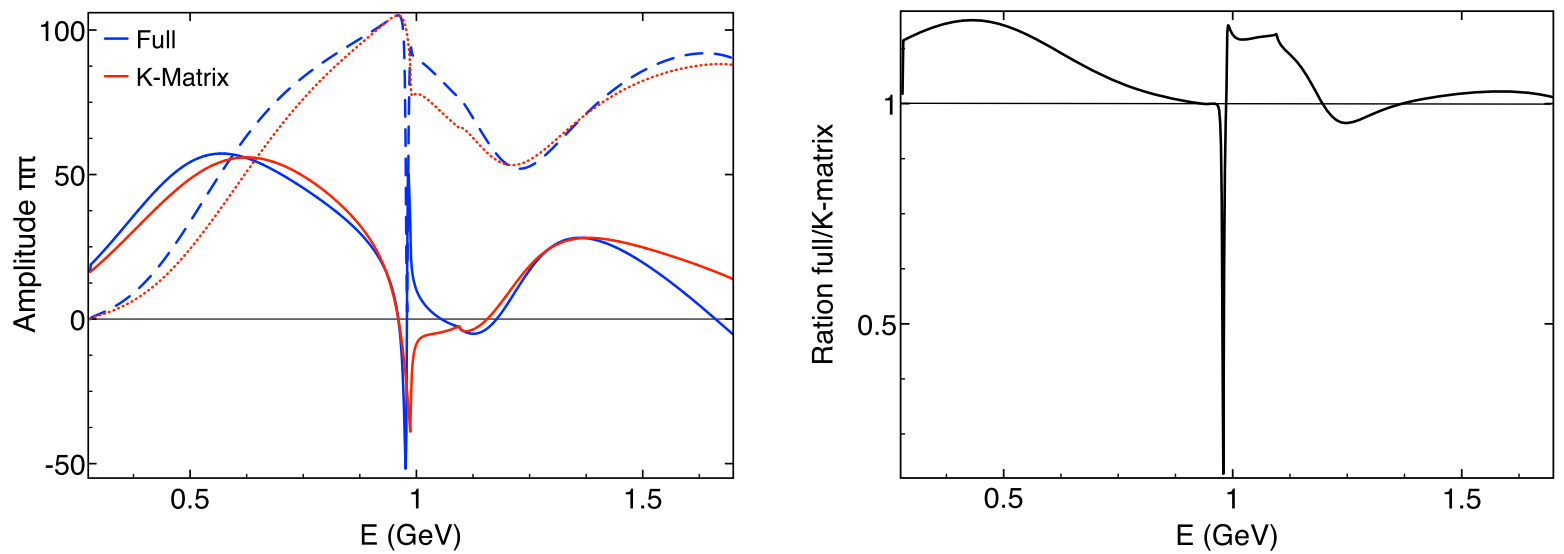

FIG. 14. Predictions for the scalar-isoscalar $\pi \pi$ amplitude with two resonances $f_{a}(1370)$ and $f_{b}(960)$, with $\epsilon=0$, superimposed to a nonresonant background from the model (58) (blue) and the $K$ matrix (red). Left: real (full curve) and imaginary (dashed curve) parts. Right: ratio of magnitudes. 

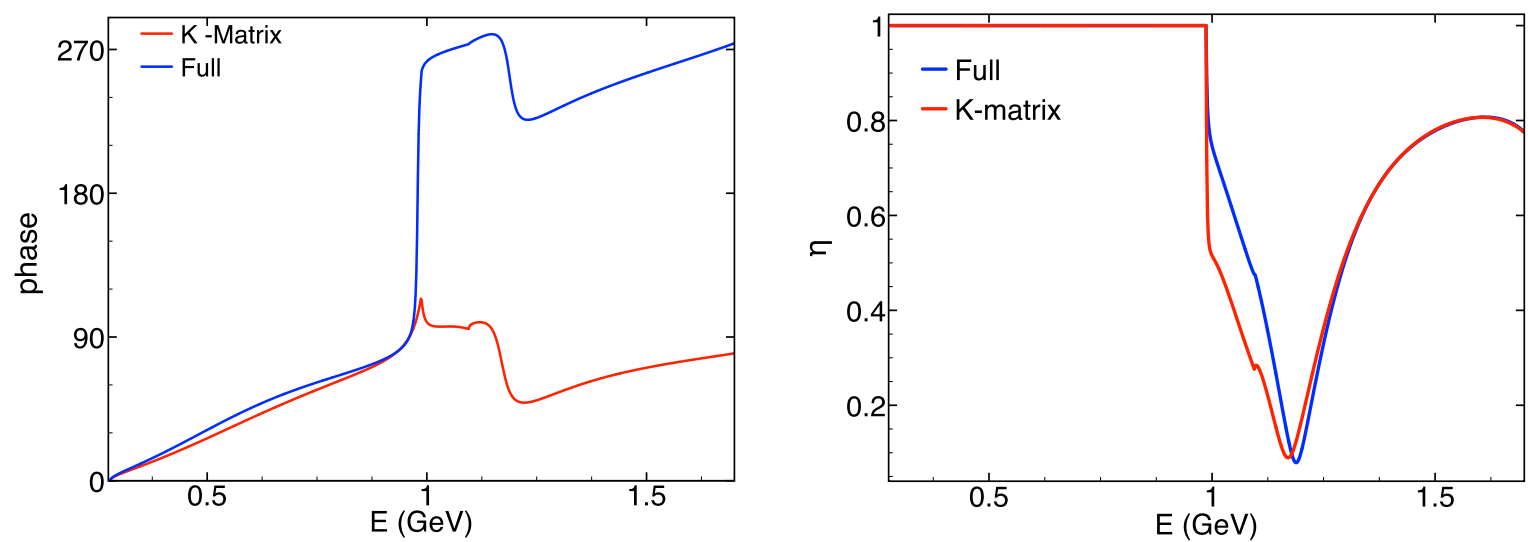

FIG. 15. Predictions for phase shifts (left) and inelasticity parameter (right) of the scalar-isoscalar $\pi \pi$ amplitude with two resonances $f_{a}(1370)$ and $f_{b}(960)$, with $\epsilon=0$, superimposed to a nonresonant background from the model (58) (blue) and the $K$ matrix (red).
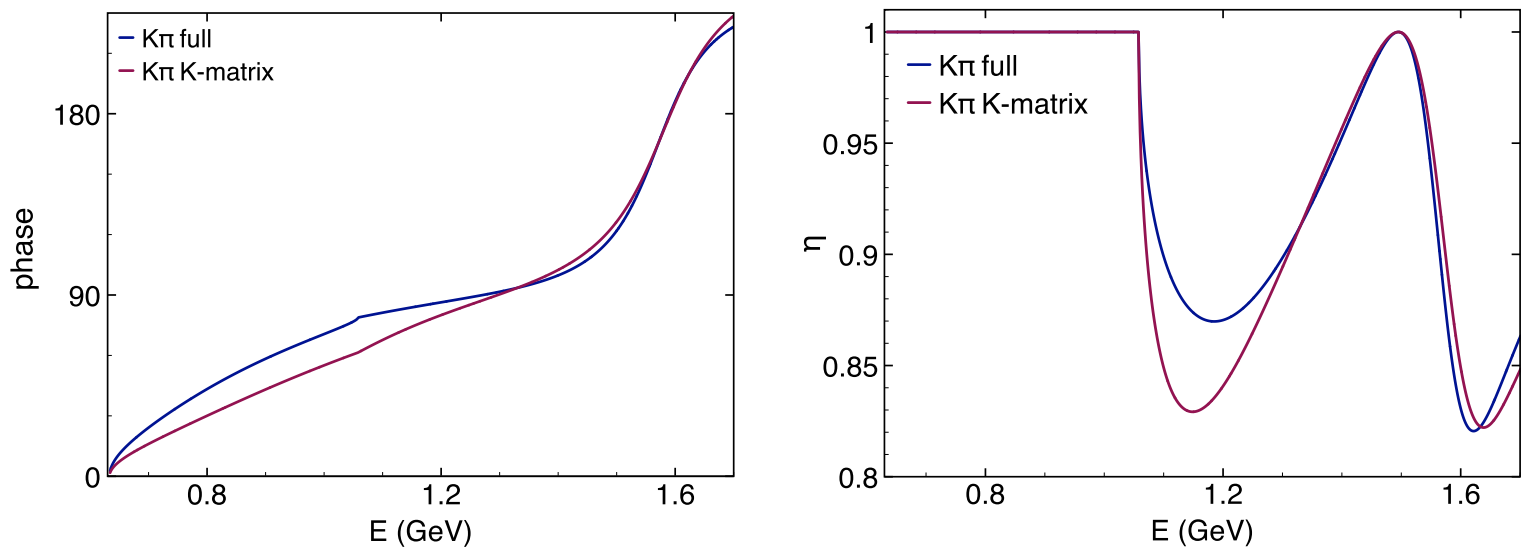

FIG. 16. Predictions for phase shifts (left) and inelasticity parameters (right) of the scalar-isovector $\pi K$ amplitude with a resonance $K_{0}^{*}$ with mass $m_{K_{0}^{*}}=1.33 \mathrm{GeV}$ superimposed to a nonresonant background from the model (C19) (dark blue) and the $K$ matrix (magenta).

$f_{b}$ in which the mechanism discussed in Sec. $\mathrm{V}$ is operating.

For the sake of completeness, in Figs. 16 and 17 we display results for phase shifts and inelasticity parameters for scalar $\pi K$ and $\pi \eta$ scatterings, predicted by Eqs. (C19) and (C17). The $\pi K$ process becomes inelastic at the $K \eta$ production threshold and includes a $K_{0}^{*}$ with mass $m_{K_{0}^{*}}=1.33 \mathrm{GeV}$, whereas the $\pi \eta$ is coupled to a $K \bar{K}$

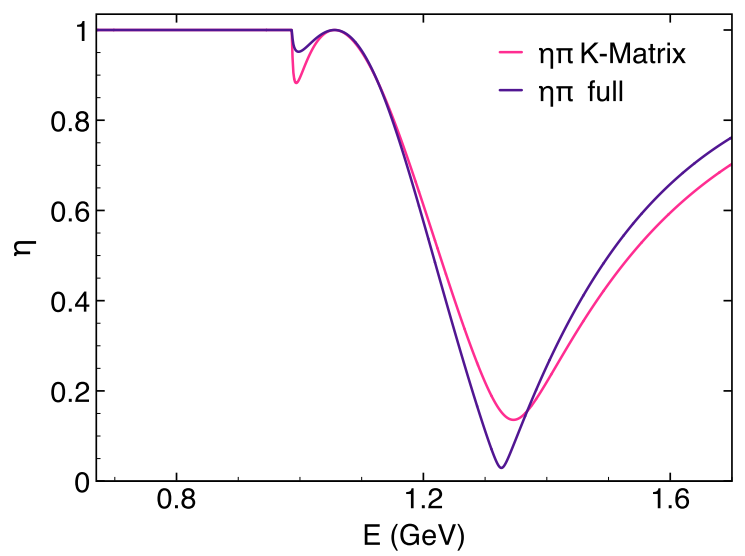

FIG. 17. Predictions for phase shifts (left) and inelasticity parameters (right) of the scalar-isovector $\pi \eta$ amplitude with a resonance $a_{0}$ with mass $m_{a_{0}}=0.95 \mathrm{GeV}$ superimposed to a nonresonant background from the model (C17) (purple) and the $K$ matrix (pink). 
through the $a_{0}$, with mass $m_{a_{0}}=0.95 \mathrm{GeV}$. Thus, in the $\pi \eta$ the resonance is below threshold and the phase passes through $90^{\circ}$ at its mass. On the other hand, in the $\pi K$ the resonance lies in the inelastic region and the influence of the background in the other channel shows up. Deviations between the model and the $K$ matrix are noticeable below $1.2 \mathrm{GeV}$ for the former and above that energy for the latter.

While inspecting the results displayed in Figs. 12-17, one should bear in mind that they rely on the coupling constants prescribed in Ref. [27] and may change significantly if other parameters are adopted.

\section{AN EXTRA RESONANCE}

The model proposed here allows for the inclusion of any number of resonances. In order to illustrate this procedure, we consider the case of an extra resonance $R^{\prime}$ in each scalar channel and begin by resorting to Eq. (59) in the case of $\pi \pi$ scattering and to Eq. (58) for $I=1 / 2$ and $I=0$. New resonances mean, of course, new masses and coupling

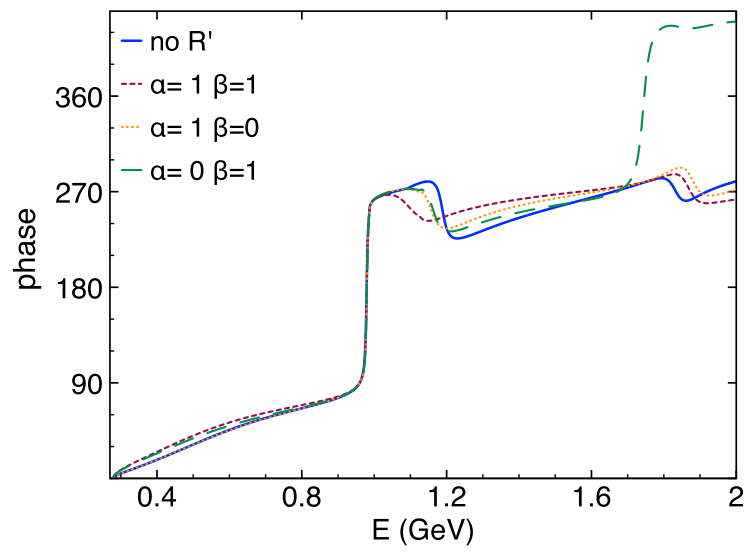

constants and, as the number of channels is large, one could have, in principle, too many new degrees of freedom to be fitted by data. In order to be conservative, we suggest that the same forms displayed after the arrows in Eqs. (B13)-(B22) be used, with

$$
\begin{aligned}
& {\left[\left(c_{d} \text { or } \tilde{c}_{d}\right)\left(s-\mathrm{mass}^{2}\right)+c_{(R \mid a b)}\right]} \\
& \quad \rightarrow\left(c_{d} \text { or } \tilde{c}_{d}\right)\left[\alpha\left(s-\mathrm{mass}^{2}\right)+\beta_{R^{\prime}} \mu^{2}\right] .
\end{aligned}
$$

In the case of the $s$-dependent couplings, this preserves the $S U$ (3) structure with a scale given by chiral perturbation theory [27], $c_{d}=0.032 \mathrm{GeV}$ and $\tilde{c}_{d}=0.018 \mathrm{GeV}$, whereas $\mu=1 \mathrm{GeV}$ is just a scale. These choices allow both $\alpha$ and $\beta$ to be dimensionless free parameters and one may guess that their values will not be far from $-1 \leq \alpha, \beta \leq 1$.

As an illustration, in Figs. 18-20 we display phase shifts and inelasticity parameters for $\pi \pi, \pi K$, and $\pi \eta$ scatterings including an extra resonance, for a choice of values of $\alpha$ and $\beta$. In all cases one notes that results do depend on the values

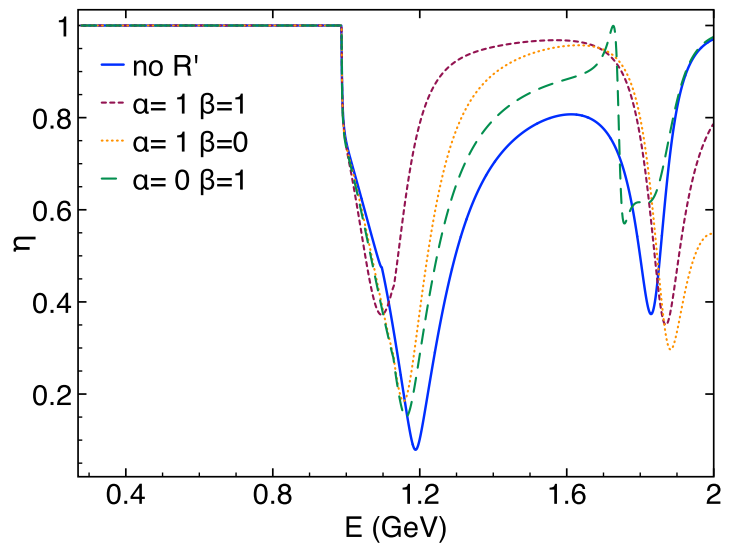

FIG. 18. Predictions for phase shifts (left) and inelasticity parameters (right) for the scalar $\pi \pi$ amplitude with an extra resonance of mass $m_{R^{\prime}}=m_{f_{0}}=1.7 \mathrm{GeV}$; the case no $R^{\prime}$ corresponds to the blue curve of Fig. 13 .
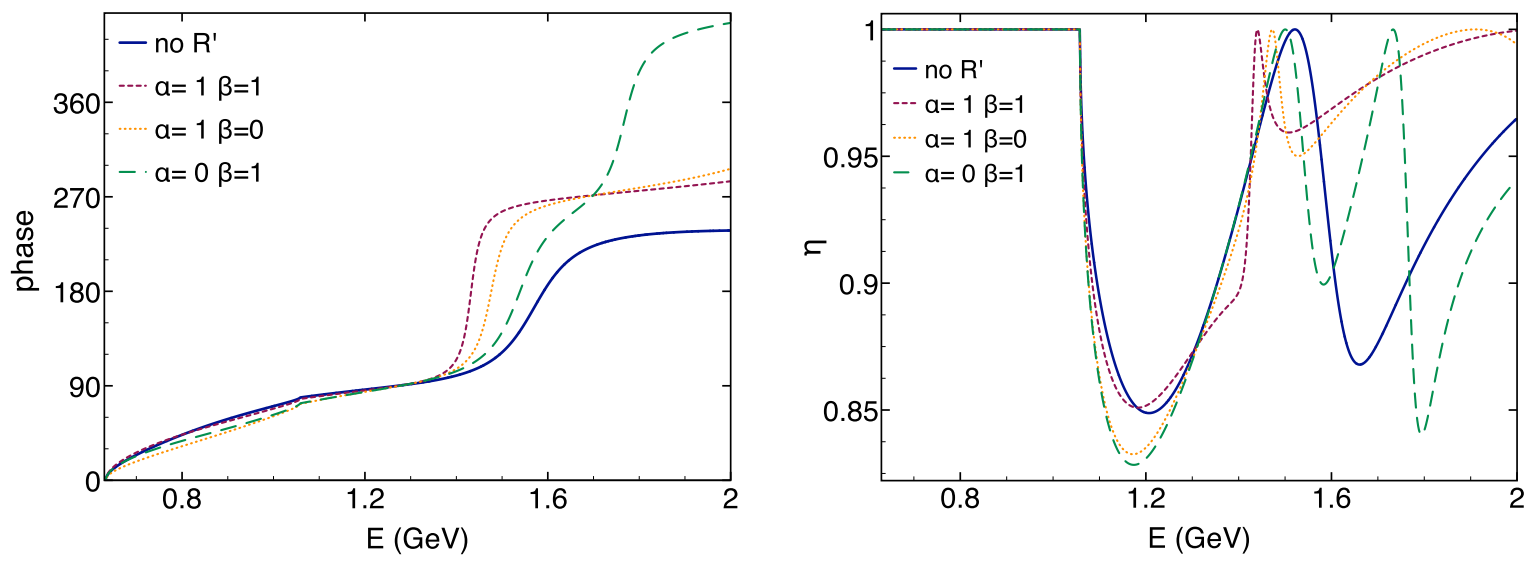

FIG. 19. Predictions for phase shifts (left) and inelasticity parameters (right) for the scalar-isovector $\pi K$ amplitude with an extra a resonance of mass $m_{R^{\prime}}=m_{K_{0}^{*}}=1.7 \mathrm{GeV}$; the case no $R^{\prime}$ corresponds to the dark blue curve of Fig. 16. 

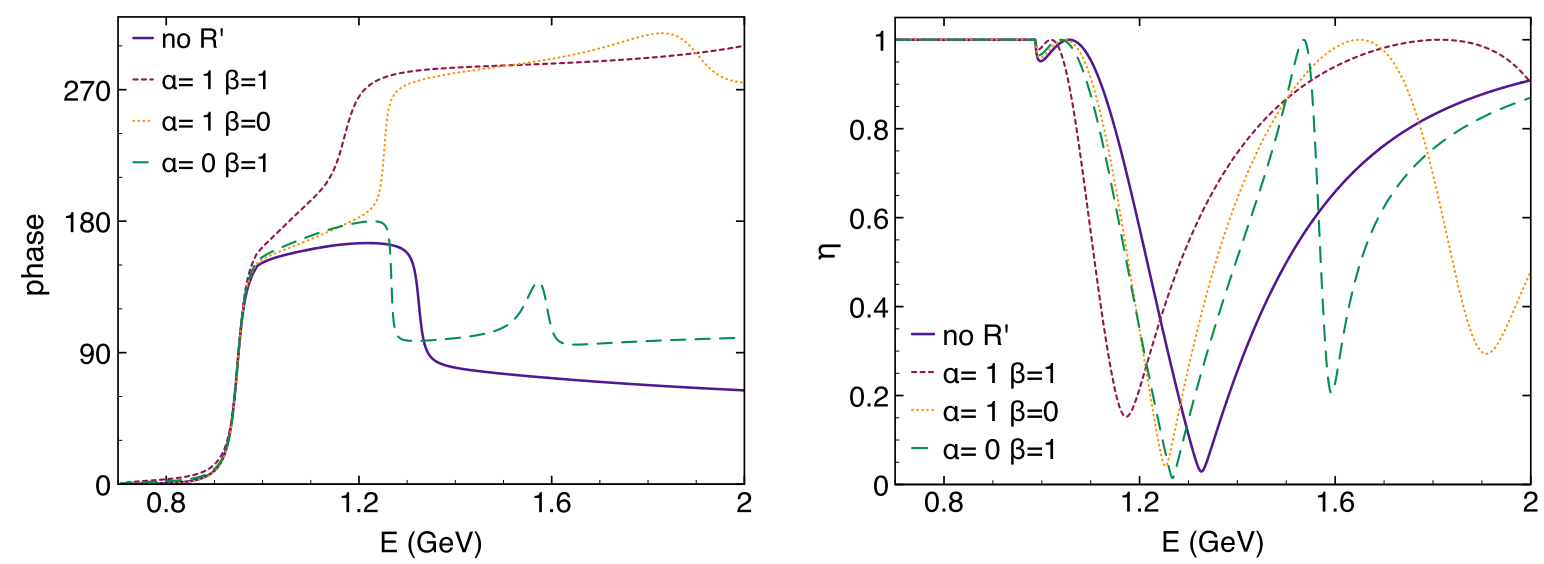

FIG. 20. Predictions for phase shifts (left) and inelasticity parameters (right) for the scalar-isovector $\pi \eta$ amplitude with an extra a resonance of mass $m_{R^{\prime}}=m_{a_{0}}=1.5 \mathrm{GeV}$; the case no $R^{\prime}$ corresponds to the purple curve of Fig. 17.

of $\alpha$ and $b$ adopted and also as expected that the highenergy regions of the curves are more sensitive to the inclusion of the extra resonance. In all cases, the extra resonance occurs in the inelastic regime and, as discussed in Sec. VA, its shape is strongly affected by a background due to channel coupling.

\section{SUMMARY AND CONCLUSIONS}

The SIM was produced more than 50 years ago and is still widely used, in spite of its many limitations. In the case of heavy-meson decays into three mesons, the model relies on the $(2+1)$ approximation, whereby strong final-state interactions involve just a two-body interacting system in the presence of a spectator. The assumption that meson-meson amplitudes are strongly dominated by resonances is essential to the model. We argued that QCD has a strong impact on this picture and that the SIM may be reliable for vector mesons in uncoupled channels but is not suited to scalar mesons. Nowadays, a proper description of low-energy meson-meson interactions requires contact with chiral perturbation theory, which implements QCD by means of effective Lagrangians. Although originally developed for low-energy processes, this theory can be reliably extended through the inclusion of resonances and unitarization techniques. In Sec. IV we have shown that the SIM and its post-QCD version give rise to rather different predictions for the scalar $\pi \pi$ amplitude, owing to both dynamics and unitarity. Another problem of the SIM concerns the coupling of channels. This effect is compulsory whenever possible, and in Sec. VA we have shown that resonances cannot be considered as dynamically isolated objects beyond coupling thresholds. This happens because pole dominance in a given channel is contaminated by background effects occurring elsewhere. Therefore, BW line shapes are unsuited for describing resonances in the inelastic regime, as shown in Sec. V B.

As an alternative to the versions employed in the SIM, in Appendix $\mathrm{C}$ we present a set of phenomenological meson-meson amplitudes in the $S U(3)$ sector, which is suitable for amplitude analyses of heavy-meson decays. Their main features include the following.

(1) Unitarization: All amplitudes are automatically unitary for energies below the first coupling threshold.

(2) Coupled channels: The treatment of coupled channels is standard and gives rise to the expected inelasticities.

(3) Dynamics: Interactions are described by chiral Lagrangians, which include both pure pseudoscalar vertices and bare resonances, with free masses and coupling constants. This ensures that chiral symmetry is obeyed at low energies and also gives rise to fitting parameters with well-defined physical meanings.

(4) Model for meson loops: Two-meson loops are an important component of scattering amplitudes. In the $s$ channel, they are given by real functions below threshold and acquire an imaginary part above it. The latter is fully determined by theory, whereas the former involve unknown renormalization constants. In Sec. VI we proposed a model for these real parts that complies with chiral symmetry and can accommodate any number of resonances.

(5) Systematic inclusion of resonances: The model can accommodate any number of resonances in each given channel.

(6) Free parameters have physical meanings: The free parameters of the model are resonance masses and constants describing their couplings to pseudoscalar mesons. Thus, their conceptual meaning is both rather conventional and process independent, whereas their empirical values can be extracted from different reactions. This allows one to envisage a situation in which one could compare various sets of values for the same parameters as determined, for instance, from chiral perturbation theory, meson-meson scattering up to $2 \mathrm{GeV}$, $D \rightarrow \pi \pi \pi, \quad D \rightarrow \pi \pi K$, and other processes. 
This would definitely promote understanding and, hopefully, much needed progress.

In this constructive approach, all imaginary terms in the amplitudes can be traced back to loops, which are also responsible for the finite widths of resonances. The parameters to be fitted are just resonance masses and coupling constants, which have a rather transparent physical meaning. As examples, we have discussed scalar amplitudes, phase shifts, and inelasticity parameters for $\pi \pi, \pi K$, and $\pi \eta$ scatterings, employing the low-energy parameters given in Ref. [27]. In all cases, results from the model for the real parts of the loop functions were compared with those from the $K$ matrix, where they are absent. One notices that the main differences occur close to the first inelastic threshold, which shows that the new model provides a clear indication for the mechanism responsible for the sharp rise observed in the $\pi \pi$ phase around $1 \mathrm{GeV}$.

\section{ACKNOWLEDGMENTS}

We would like to thank Jonas Rademacker and José Ramon Pelaez for fruitful discussions. P. C. M. was supported by Marie Sklodowska-Curie (MSCA) action (European Union program Horizon 2020) Grant No. 799974.

\section{APPENDIX A: TWO-MESON PROPAGATORS AND FUNCTIONS $\Omega$}

The conventional expressions presented here are displayed for the sake of completeness and rely on results from Ref. [26]. These integrals do not include symmetry factors, which are accounted for in the main text. One deals with both $S$ and $P$ waves and the corresponding two-meson propagators are associated with

$$
\begin{gathered}
\left\{I_{a b} ; I_{a b}^{\mu \nu}\right\}=\int \frac{d^{4} \ell}{(2 \pi)^{4}} \frac{\left\{1 ; \ell^{\mu} \ell^{\nu}\right\}}{D_{a} D_{b}}, \\
D_{a}=(\ell+p / 2)^{2}-M_{a}^{2}, \quad D_{b}=(\ell-p / 2)^{2}-M_{b}^{2},
\end{gathered}
$$

where $p^{2}=s$ and both integrals are evaluated using dimensional-regularization techniques. The function $I_{a b}$ reads

$$
I_{a b}=i \frac{1}{16 \pi^{2}}\left[\Lambda_{a b}+\Pi_{a b}\right]
$$

where $\Lambda_{a b}$ is a function of the renormalization scale $\mu$ and the number of dimensions $n$, which diverges in the limit $n \rightarrow 4$, whereas $\Pi$ is a regular component, given by

$$
\begin{gathered}
s<\left(M_{a}-M_{b}\right)^{2} \rightarrow \Pi_{a b} \\
=\Pi_{a b}^{0}+\frac{\sqrt{\lambda}}{s} \ln \left[\frac{\left.M_{a}^{2}+M_{b}^{2}-s+\sqrt{\lambda}\right]}{2 M_{a} M_{b}}\right], \quad \text { (A4) } \\
\left(M_{a}-M_{b}\right)^{2}<s<\left(M_{a}^{2}+M_{b}^{2}\right) \rightarrow \Pi_{a b} \\
=\Pi_{a b}^{0}-\frac{\sqrt{-\lambda}}{s} \tan ^{-1}\left[\frac{\sqrt{-\lambda}}{M_{a}^{2}+M_{b}^{2}-s}\right], \quad(\mathrm{A} 5) \\
\left(M_{a}^{2}+M_{b}^{2}\right)<s<\left(M_{a}+M_{b}\right)^{2} \rightarrow \Pi_{a b} \\
=\Pi_{a b}^{0}-\frac{\sqrt{-\lambda}}{s}\left\{\tan ^{-1}\left[\frac{\sqrt{-\lambda}}{M_{a}^{2}+M_{b}^{2}-s}\right]+\pi\right\}, \\
s>\left(M_{a}+M_{b}\right)^{2} \rightarrow \Pi_{a b} \\
=\Pi_{a b}^{0}-\frac{\sqrt{\lambda}}{s} \ln \left[\frac{\left.s-M_{a}^{2}-M_{b}^{2}+\sqrt{\lambda}\right]+i \pi \frac{\sqrt{\lambda}}{2 M_{a} M_{b}},}{\lambda}\right. \\
\Pi_{a b}^{0}=1+\frac{M_{a}^{2}+M_{b}^{2}}{M_{a}^{2}-M_{b}^{2}} \ln \left[\frac{M_{a}}{M_{b}}\right]-\frac{M_{a}^{2}-M_{b}^{2}}{s} \ln \left[\frac{M_{a}}{M_{b}}\right], \\
\lambda=s^{2}-2 s\left(M_{a}^{2}+M_{b}^{2}\right)+\left(M_{a}^{2}-M_{b}^{2}\right)^{2} .
\end{gathered}
$$

For $M_{a}=M_{b}, \Pi_{a a}^{0}=2$. The tensor integral is

$$
\begin{aligned}
I_{a b}^{\mu \nu}= & i \frac{1}{16 \pi^{2}}\left\{\left[\frac{p^{\mu} p^{\nu}}{s} \Lambda_{a b}^{p p}-g^{\mu \nu} \Lambda_{a b}^{g}\right]\right. \\
& \left.+\left[\frac{p^{\mu} p^{\nu}}{s}-g^{\mu \nu}\right] \frac{\lambda}{12 s} \Pi_{a b}\right\},
\end{aligned}
$$

where $\Lambda_{a b}^{p p}$ and $\Lambda_{a b}^{g}$ are divergent quantities.

In the calculation of final-state interactions, it is more convenient to use the functions $\Omega$, defined from the regular parts of Eqs. (A3) and (A10) as

$\Omega_{a b}^{S}=i\left[\right.$ regular part of $\left.I_{a b}\right] \rightarrow \Omega_{a b}^{S}=-\frac{1}{16 \pi^{2}} \Pi_{a b}$,

$$
\begin{aligned}
\frac{1}{4}\left[\frac{p^{\mu} p^{\mu}}{s}-g^{\mu \nu}\right] \Omega_{a b}^{P} & =i\left[\text { regular part of } I_{a b}^{\mu \nu}\right] \rightarrow \Omega_{a b}^{P} \\
& =-\frac{\lambda}{48 \pi^{2} s} \Pi_{a b} .
\end{aligned}
$$

As indicated in Eqs. (A4)-(A9), the functions $\Omega$ are real below the threshold at $s_{\text {th }}=\left(M_{a}+M_{b}\right)^{2}$ and acquire an imaginary component above it. This imaginary part is not affected by infinities and is a well-defined prediction of the theory that is necessary to implement unitarity. 
In the c.m. frame the momentum $Q_{a b}$ is given by

$$
\begin{aligned}
Q_{a b} & =\frac{\sqrt{\lambda}}{2 \sqrt{s}} \\
& =\frac{1}{2} \sqrt{s-2\left(M_{a}^{2}+M_{b}^{2}\right)+\left(M_{a}^{2}-M_{b}^{2}\right)^{2} / s}
\end{aligned}
$$

and the imaginary components read

$$
\begin{aligned}
& {\left[\Omega_{a b}^{S}\right]^{I}=-\frac{1}{8 \pi} \frac{Q_{a b}}{\sqrt{s}} \theta\left(s-\left(M_{a}+M_{b}\right)^{2}\right),} \\
& {\left[\Omega_{a b}^{P}\right]^{I}=-\frac{1}{6 \pi} \frac{Q_{a b}^{3}}{\sqrt{s}} \theta\left(s-\left(M_{a}+M_{b}\right)^{2}\right),}
\end{aligned}
$$

where $\theta$ is the Heaviside step function.

\section{APPENDIX B: SCATTERING KERNELS}

We consider scattering amplitudes that can have $S U(3)$ resonances as intermediate states. They depend on interaction kernels for channels with angular momentum $J=1$, 0 and isospin $I=1,1 / 2,0$. All kernels are written as sums of a LO chiral polynomial and NLO resonance contributions [27]. In the resonance sector, we consider the standard $S U$ (3) contributions, supplemented by an extra term $R^{(J, I)}$ for each channel, with free masses and coupling constants, denoted by a prime. The usual Mandelstam variables are $s, t, u$ and the kernels $\mathcal{K}_{a b \rightarrow c d}^{(J, I)}$ for the process $P_{a} P_{b} \rightarrow P_{c} P_{d}$ are as follows.

Vector sector: In the case $J=1$, kernels are written without a factor $\left[2 t+s-2\left(M_{a}^{2}+M_{b}^{2}\right)+\right.$ $\left.\left(M_{a}^{2}-M_{b}^{2}\right)^{2} / s\right]$, which becomes $(t-u)$ in the case of identical particles and reduces to $\left[4 Q^{2} \cos \theta\right]$ in the center-of-mass frame.

isospin $I=1$ :

$$
\begin{gathered}
\mathcal{K}_{(\pi \pi \mid \pi \pi)}^{(1,1)}=\frac{1}{F^{2}}-\frac{s G_{(\rho \mid \pi \pi)}^{2}}{s-m_{\rho}^{2}}-\frac{s G_{\left(\rho^{\prime} \mid \pi \pi\right)}^{2}}{s-m_{\rho^{\prime}}^{2}}, \\
\mathcal{K}_{(\pi \pi \mid K K)}^{(1,1)}=\frac{\sqrt{2}}{2 F^{2}}-\frac{s G_{(\rho \mid \pi \pi)} G_{(\rho \mid K K)}}{s-m_{\rho}^{2}} \\
-\frac{s G_{\left(\rho^{\prime} \mid \pi \pi\right)} G_{\left(\rho^{\prime} \mid K K\right)}}{s-m_{\rho^{\prime}}^{2}}, \\
\mathcal{K}_{(K K \mid K K)}^{(1,1)}=\frac{1}{2 F^{2}}-\frac{s G_{(\rho \mid K K)}^{2}}{s-m_{\rho}^{2}}-\frac{s G_{\left(\rho^{\prime} \mid K K\right)}^{2}}{s-m_{\rho^{\prime}}^{2}} \\
G_{(\rho \mid \pi \pi)}=\frac{\sqrt{2} G_{V}}{F^{2}}, \\
G_{(\rho \mid K K)}=\frac{G_{V}}{F^{2}} .
\end{gathered}
$$

In the framework of resonance ChPT (RChPT), $G_{V}$ lies in the range 53-69 MeV. Of special interest is the relationship $G_{V}=F / \sqrt{2} \simeq 66 \mathrm{MeV}$, associated with vector-meson dominance [27].

isospin $I=1 / 2$ :

$$
\begin{aligned}
\mathcal{K}_{(\pi K \mid \pi K)}^{(1,1 / 2)}= & \frac{3}{8 F^{2}}-\frac{s G_{\left(K^{*} \mid \pi K\right)}^{2}}{s-m_{K^{*}}^{2}}-\frac{s G_{\left(K^{*^{\prime}} \mid \pi K\right)}^{2}}{s-m_{K^{*^{\prime}}}^{2}}, \\
\mathcal{K}_{(\pi K \mid 8 K)}^{(1,1 / 2)}= & \frac{3}{8 F^{2}}-\frac{s G_{\left(K^{*} \mid \pi K\right)} G_{\left(K^{*} \mid 8 K\right)}}{s-m_{K^{*}}^{2}} \\
& -\frac{s G_{\left(K^{*^{\prime}} \mid \pi K\right)} G_{\left(K^{*^{\prime}} \mid 8 K\right)}}{s-m_{K^{*^{\prime}}}^{2}},
\end{aligned}
$$

$$
\begin{gathered}
\mathcal{K}_{(K 8 \mid K 8)}^{(1,1 / 2)}=\frac{3}{8 F^{2}}-\frac{s G_{\left(K^{*} \mid 8 K\right)}^{2}}{s-m_{K^{*}}^{2}}-\frac{s G_{\left(K^{*^{\prime}} \mid 8 K\right)}^{2}}{s-m_{K^{*^{\prime}}}^{2}}, \\
G_{\left(K^{*} \mid \pi K\right)}=\frac{\sqrt{3} G_{V}}{2 F^{2}} . \\
G_{\left(K^{*} \mid 8 K\right)}=-\frac{\sqrt{3} G_{V}}{2 F^{2}} .
\end{gathered}
$$

isospin $I=0$ :

$$
\begin{gathered}
\mathcal{K}_{(K K \mid K K)}^{(1,0)}=\frac{3}{2 F^{2}}-\frac{s G_{(\phi \mid K K)}^{2}}{s-m_{\phi}^{2}}-\frac{s G_{\left(\phi^{\prime} \mid K K\right)}^{2}}{s-m_{\phi^{\prime}}^{2}}, \\
G_{(\phi \mid K K)}=\frac{\sqrt{3} G_{V} \sin \theta}{F^{2}} .
\end{gathered}
$$

In a previous work [10] we considered a dressed $\phi$ propagator, which accounts for the partial width of the decay $\phi \rightarrow(\rho \pi+\pi \pi \pi)$. This small contribution is technically involved and here we ignore it for the sake of simplicity. The partial width for $\phi \rightarrow K \bar{K}$ yields [33] $\sin \theta=0.605$.

Scalar sector: Chiral perturbation theory accurately predicts how $S U(3)$-breaking effects, characterized by pseudoscalar masses, influence low-energy observables. The couplings of scalar resonances to two pseudoscalars involve energy-dependent factors which conserve $S U(3)$, associated with the constants $c_{d}$ and $\tilde{c}_{d}$, supplemented by symmetry-breaking terms, proportional to $c_{m}$ and $\tilde{c}_{m}$. In this work we need to extend scattering amplitudes up to energies well beyond the $\rho$ mass, which is the upper bound for ChPT, and therefore we keep the $S U(3)$-invariant parts of scalar-two-pseudoscalar couplings and allow the symmetry-breaking parts to be described by phenomenological parameters $c$. Below we denote the resonances by $a_{0} \rightarrow$ $(J, I=0,1), K_{0}^{*} \rightarrow(J=0,1 / 2)$, So $\rightarrow(J, I=0,0)$ octet, $S 1 \rightarrow(J, I=0,0)$ singlet and list these couplings using the 


$$
\begin{aligned}
& G_{\left(a_{0} \mid \pi 8\right)}=\frac{2}{\sqrt{3} F^{2}}\left[c_{d}\left(s-M_{\pi}^{2}-M_{8}^{2}\right)+c_{m} 2 M_{\pi}^{2}\right] \\
& \rightarrow \frac{2}{\sqrt{3} F^{2}}\left[c_{d}\left(s-M_{\pi}^{2}-M_{8}^{2}\right)+c_{\left(a_{0} \mid \pi 8\right)}\right], \\
& G_{(S 1 \mid K K)}=\frac{4}{F^{2}}\left[\tilde{c}_{d} s-\left(\tilde{c}_{d}-\tilde{c}_{m}\right) 2 M_{K}^{2}\right] \\
& \rightarrow \frac{4}{F^{2}}\left[\tilde{c}_{d}\left(s-2 M_{K}^{2}\right)+c_{(S 1 \mid K K)}\right], \\
& G_{(S 1 \mid 88)}=\frac{2}{F^{2}}\left[\tilde{c}_{d} s-\left(\tilde{c}_{d}-\tilde{c}_{m}\right) 2 M_{8}^{2}\right] \\
& \rightarrow \frac{2}{F^{2}}\left[\tilde{c}_{d}\left(s-2 M_{8}^{2}\right)+c_{(S 1 \mid 88)}\right] .
\end{aligned}
$$$$
G_{\left(a_{0} \mid K K\right)}=\frac{\sqrt{2}}{F^{2}}\left[c_{d} s-\left(c_{d}-c_{m}\right) 2 M_{K}^{2}\right]
$$$$
\rightarrow \frac{\sqrt{2}}{F^{2}}\left[c_{d}\left(s-2 M_{K}^{2}\right)+c_{\left(a_{0} \mid K K\right)}\right],
$$$$
G_{\left(K_{0}^{*} \mid \pi K\right)}=\frac{\sqrt{3}}{\sqrt{2} F^{2}}\left[c_{d} s-\left(c_{d}-c_{m}\right)\left(M_{\pi}^{2}+M_{K}^{2}\right)\right]
$$$$
\rightarrow \frac{\sqrt{3}}{\sqrt{2} F^{2}}\left[c_{d}\left(s-M_{\pi}^{2}-M_{K}^{2}\right)+c_{\left(K_{0}^{*} \mid \pi 8\right)}\right],
$$$$
G_{\left(K_{0}^{*} \mid K 8\right)}=-\frac{1}{\sqrt{6} F^{2}}\left[c_{d}\left(s-M_{K}^{2}-M_{8}^{2}\right)\right.
$$$$
\left.+c_{m}\left(-8 M_{\pi}^{2}+11 M_{K}^{2}+3 M_{8}^{2}\right) / 3\right]
$$$$
\rightarrow-\frac{1}{\sqrt{6} F^{2}}\left[c_{d}\left(s-M_{\pi}^{2}-M_{8}^{2}\right)\right.
$$$$
+c_{\left(K_{0}^{*} \mid K 8\right)} \text {, }
$$$$
G_{(S o \mid \pi \pi)}=-\frac{\sqrt{2}}{F^{2}}\left[c_{d} s-\left(c_{d}-c_{m}\right) 2 M_{\pi}^{2}\right]
$$$$
\rightarrow-\frac{\sqrt{2}}{F^{2}}\left[c_{d}\left(s-2 M_{\pi}^{2}\right)+c_{(S o \mid \pi \pi)}\right],
$$$$
G_{(S o \mid K K)}=\frac{\sqrt{6}}{3 F^{2}}\left[c_{d} s-\left(c_{d}-c_{m}\right) 2 M_{K}^{2}\right]
$$$$
\rightarrow \frac{\sqrt{6}}{3 F^{2}}\left[c_{d}\left(s-2 M_{K}^{2}\right)+c_{(S o \mid K K)}\right],
$$$$
G_{(S o \mid 88)}=\frac{\sqrt{6}}{3 F^{2}}\left[c_{d}\left(s-2 M_{8}^{2}\right)+c_{m}\left(16 M_{K}^{2}-10 M_{\pi}^{2}\right) / 3\right]
$$$$
\rightarrow \frac{\sqrt{6}}{3 F^{2}}\left[c_{d}\left(s-2 M_{8}^{2}\right)+c_{(S o \mid 88)}\right],
$$$$
G_{(S 1 \mid \pi \pi)}=\frac{2 \sqrt{3}}{F^{2}}\left[\tilde{c}_{d} s-\left(\tilde{c}_{d}-\tilde{c}_{m}\right) 2 M_{\pi}^{2}\right]
$$$$
\rightarrow \frac{2 \sqrt{3}}{F^{2}}\left[\tilde{c}_{d}\left(s-2 M_{\pi}^{2}\right)+c_{(S 1 \mid \pi \pi)}\right],
$$

In RChPT [27], one has $\left|c_{d}\right|=0.032 \mathrm{MeV},\left|c_{m}\right|=$ $0.042 \mathrm{MeV},\left|\tilde{c}_{d}\right|=\left|c_{d}\right| / \sqrt{3}$, and $\left|\tilde{c}_{m}\right|=\left|c_{m}\right| / \sqrt{3}$. isospin $I=1$ :

$$
\begin{gathered}
\mathcal{K}_{(\pi 8 \mid \pi 8)}^{(0,1)}=\frac{2 M_{\pi}^{2}}{3 F^{2}}-\frac{G_{\left(a_{0} \mid \pi 8\right)}^{2}}{s-m_{a_{0}}^{2}}-\frac{G_{\left(a_{0}^{\prime} \mid \pi 8\right)}^{2}}{s-m_{a_{0}^{\prime}}{ }^{2}}, \\
\mathcal{K}_{(\pi 8 \mid K K)}^{(0,1)}=\frac{\left(3 s-4 M_{K}^{2}\right)}{\sqrt{6} F^{2}}-\frac{G_{\left(a_{0} \mid \pi 8\right)} G_{\left(a_{0} \mid K K\right)}}{s-m_{a_{0}}^{2}} \\
-\frac{G_{\left(a_{0}^{\prime} \mid \pi 8\right)} G_{\left(a_{0}^{\prime} \mid K K\right)}}{s-m_{a_{0}^{\prime}}{ }^{2}}, \\
\mathcal{K}_{(K K \mid K K)}^{(0,1)}=\frac{s}{2 F^{2}}-\frac{G_{\left(a_{0} \mid K K\right)}^{2}}{s-m_{a_{0}}^{2}}-\frac{G_{\left(a_{0}^{\prime} \mid K K\right)}^{2}}{s-m_{a_{0}^{\prime}}{ }^{2}}
\end{gathered}
$$

isospin $I=1 / 2$ :

$$
\begin{aligned}
\mathcal{K}_{(\pi K \mid \pi K)}^{(0,1 / 2)} & -\frac{1}{8 F^{2}}\left[5 s-2\left(M_{\pi}^{2}+M_{K}^{2}\right)+\frac{3\left(M_{\pi}^{2}+M_{K}^{2}\right)^{2}}{s}\right], \\
& -\frac{G_{\left(K_{0}^{*} \mid \pi K\right)}^{2}}{s-m_{K_{0}^{*}}^{2}}-\frac{G_{\left(K_{0}^{*^{\prime}} \mid \pi K\right)}^{2}}{s-m_{K_{0}^{\prime^{\prime}}}{ }^{2}} . \\
\mathcal{K}_{(\pi K \mid 8 K)}^{(0,1 / 2)}= & -\frac{1}{24 F^{2}}\left[9 s-16 M_{\pi}^{2}-8 M_{K}^{2}\right. \\
& \left.+6 M_{8}^{2}+\frac{9\left(M_{\pi}^{2}+M_{K}^{2}\right)^{2}}{s}\right], \\
& -\frac{G_{\left(K_{0}^{*} \mid \pi K\right)} G_{\left(K_{0}^{*} \mid 8 K\right)}-\frac{G_{\left(K_{0}^{*^{\prime}} \mid \pi K\right)} G_{\left(K_{0}^{*^{\prime}} \mid 8 K\right)}}{s-m_{K_{0}^{*}}^{2}} .}{s-m_{K_{0}^{\prime^{\prime}}}{ }^{2}} .
\end{aligned}
$$




$$
\begin{aligned}
\mathcal{K}_{(8 K \mid 8 K)}^{(0,1 / 2)}= & -\frac{1}{24 F^{2}}\left[9 s+4 M_{\pi}^{2}-18 M_{K}^{2}\right. \\
& \left.+3 M_{8}^{2}+\frac{9\left(M_{K}^{2}+M_{8}^{2}\right)^{2}}{s}\right], \\
& -\frac{G_{\left(K_{0}^{*} \mid 8 K\right)}^{2}}{s-m_{K_{0}^{*}}^{2}}-\frac{G_{\left(K_{0}^{*^{\prime}} \mid 8 K\right)}^{2}}{s-m_{K_{0}^{*^{\prime}}}^{2}}
\end{aligned}
$$

isospin $I=0$ :

We allow for the possibility that the first two observed resonances in this channel, denoted by $f_{a}$ and $f_{b}$, can be mixtures of octet and singlet states $S o$ and $S 1$. The mixing angle $\epsilon$ is defined by

$$
\begin{gathered}
\left|f_{a}\right\rangle=\cos \epsilon|S 1\rangle+\sin \epsilon|S o\rangle, \\
\left|f_{b}\right\rangle=-\sin \epsilon|S 1\rangle+\cos \epsilon|S o\rangle
\end{gathered}
$$

and the kernels read

$$
\begin{aligned}
& \mathcal{K}_{(\pi \pi \mid \pi \pi)}^{(0,0)}=\frac{\left(2 s-M_{\pi}^{2}\right)}{F^{2}}-\frac{G_{\left(f_{a}|\pi \pi| \pi \pi\right)}}{s-m_{f_{a}}^{2}} \\
& -\frac{G_{\left(f_{b}|\pi \pi| \pi \pi\right)}}{s-m_{f_{b}}^{2}}-\frac{G_{\left(f^{\prime} \mid \pi \pi\right)}^{2}}{s-m_{f^{\prime}}^{2}}, \\
& \mathcal{K}_{(\pi \pi \mid K K)}^{(0,0)}=\frac{\sqrt{3} s}{2 F^{2}}-\frac{G_{\left(f_{a}|\pi \pi| K K\right)}}{s-m_{f_{a}}^{2}}-\frac{G_{\left(f_{b}|\pi \pi| K K\right)}}{s-m_{f_{b}}^{2}} \\
& -\frac{G_{\left(f^{\prime} \mid \pi \pi\right)} G_{\left(f^{\prime} \mid K K\right)}}{s-m_{f^{\prime}}^{2}}, \\
& \mathcal{K}_{(\pi \pi \mid 88)}^{(0,0)}=\frac{\sqrt{3} M_{\pi}^{2}}{3 F^{2}}-\frac{G_{\left(f_{a}|\pi \pi| 88\right)}}{s-m_{f_{a}}^{2}}-\frac{G_{\left(f_{b}|\pi \pi| 88\right)}}{s-m_{f_{b}}^{2}} \\
& -\frac{G_{\left(f^{\prime} \mid \pi \pi\right)} G_{\left(f^{\prime} \mid 88\right)}}{s-m_{f^{\prime}}^{2}}, \\
& \mathcal{K}_{(K K \mid K K)}^{(0,0)}=\frac{3 s}{2 F^{2}}-\frac{G_{\left(f_{a}|K K| K K\right)}}{s-m_{f_{a}}^{2}} \\
& -\frac{G_{\left(f_{b}|K K| K K\right)}}{s-m_{f_{b}}^{2}}-\frac{G_{\left(f^{\prime} \mid K K\right)}^{2}}{s-m_{f^{\prime}}^{2}}, \\
& \mathcal{K}_{(K K \mid 88)}^{(0,0)}=\frac{\left(9 s-8 M_{K}^{2}\right)}{6 F^{2}}-\frac{G_{\left(f_{a}|K K| 88\right)}}{s-m_{f_{a}}^{2}} \\
& -\frac{G_{\left(f_{b}|K K| 88\right)}}{s-m_{f_{b}}^{2}}-\frac{G_{\left(f^{\prime} \mid K K\right)} G_{\left(f^{\prime} \mid 88\right)}}{s-m_{f^{\prime}}^{2}}, \\
& \mathcal{K}_{(88 \mid 88)}^{(0,0)}=\frac{\left(-7 M_{\pi}^{2}+16 M_{K}^{2}\right)}{9 F^{2}}-\frac{G_{\left(f_{a}|88| 88\right)}}{s-m_{f_{a}}^{2}} \\
& -\frac{G_{\left(f_{b}|88| 88\right)}}{s-m_{f_{b}}^{2}}-\frac{G_{\left(f^{\prime} \mid 88\right)}^{2}}{s-m_{f^{\prime}}^{2}},
\end{aligned}
$$

with

$$
\begin{aligned}
& G_{\left(f_{a}|\pi \pi| \pi \pi\right)}=\sin ^{2} \epsilon G_{(S o \mid \pi \pi)}^{2}+\cos ^{2} \epsilon G_{(S 1 \mid \pi \pi)}^{2}, \\
& G_{\left(f_{a}|\pi \pi| K K\right)}=\sin ^{2} \epsilon G_{(S o \mid \pi \pi)} G_{(S o \mid K K)} \\
& +\cos ^{2} \epsilon G_{(S 1 \mid \pi \pi)} G_{(S 1 \mid K K)}, \\
& G_{\left(f_{a}|\pi \pi| 88\right)}=\sin ^{2} \epsilon G_{(S o \mid \pi \pi)} G_{(S o \mid 88)} \\
& +\cos ^{2} \epsilon G_{(S 1 \mid \pi \pi)} G_{(S 1 \mid 88)}, \\
& G_{\left(f_{a}|K K| K K\right)}=\sin ^{2} \epsilon G_{(S o \mid K K)}^{2}+\cos ^{2} \epsilon G_{(S 1 \mid K K)}^{2}, \\
& G_{\left(f_{a}|K K| 88\right)}=\sin ^{2} \epsilon G_{(S o \mid K K)} G_{(S o \mid 88)} \\
& +\cos ^{2} \epsilon G_{(S 1 \mid K K)}^{2} G_{(S 1 \mid 88)}, \\
& G_{\left(f_{a}|88| 88\right)}=\sin ^{2} \epsilon G_{(S o \mid 88)}^{2}+\cos ^{2} \epsilon G_{(S 1 \mid 88)}^{2}, \\
& G_{\left(f_{b}|\pi \pi| \pi \pi\right)}=\cos ^{2} \epsilon G_{(S o \mid \pi \pi)}^{2}+\sin ^{2} \epsilon G_{(S 1 \mid \pi \pi)}^{2}, \\
& G_{\left(f_{b}|\pi \pi| K K\right)}=\cos ^{2} \epsilon G_{(S o \mid \pi \pi)} G_{(S o \mid K K)} \\
& +\sin ^{2} \epsilon G_{(S 1 \mid \pi \pi)} G_{(S 1 \mid K K)}, \\
& G_{\left(f_{b}|\pi \pi| 88\right)}=\cos ^{2} \epsilon G_{(S o \mid \pi \pi)} G_{(S o \mid 88)} \\
& +\sin ^{2} \epsilon G_{(S 1 \mid \pi \pi)} G_{(S 1 \mid 88)}, \\
& G_{\left(f_{b}|K K| K K\right)}=\cos ^{2} \epsilon G_{(S o \mid K K)}^{2}+\sin ^{2} \epsilon G_{(S 1 \mid K K)}^{2}, \\
& G_{\left(f_{b}|K K| 88\right)}=\cos ^{2} \epsilon G_{(S o \mid K K)} G_{(S o \mid 88)} \\
& +\sin ^{2} \epsilon G_{(S 1 \mid K K)} G_{(S 1 \mid 88)}, \\
& G_{\left(f_{b}|88| 88\right)}=\cos ^{2} \epsilon G_{(S o \mid 88)}^{2}+\sin ^{2} \epsilon G_{(S 1 \mid 88)}^{2} .
\end{aligned}
$$

\section{APPENDIX C: COUPLED-CHANNEL SCATTERING AMPLITUDES}

In the discussion of schematic dynamics in the main text, we show that the scattering amplitudes for pseudoscalars have the general form given by Eqs. (3)-(5), reproduced below:

$$
\begin{aligned}
& A=\mathcal{K} \times\left[1+(\text { loop } \times \mathcal{K})+(\text { loop } \times \mathcal{K})^{2}+(\text { loop } \times \mathcal{K})^{3}+\cdots\right], \\
& \quad(\text { loop })=\text { real part }+i \Omega^{I} \\
& \mathcal{K}=\mathcal{K}_{0}+\mathcal{K}_{1}+\mathcal{K}_{2}+\cdots
\end{aligned}
$$

where the functions (loop) involve the $\Omega$ discussed in Appendix $\mathrm{A}$ and the kernels $\mathcal{K}_{0}$ are given in Appendix B. Here we present the scattering amplitudes for the process $P_{k} P_{\ell} \rightarrow P_{a} P_{b}$ in the coupled-channel formalism. It is important to stress that, although expressed in terms of 
$\Omega$ and $\mathcal{K}$, the results displayed are quite general and fully independent of the specific forms chosen for these functions. They just rely on the well-established techniques for dealing with coupled-channel problems.

The factor $($ loop $\times \mathcal{K})$ corresponds to mixing matrix elements $M^{(J, I)}$, which are given by [10]

$$
\begin{aligned}
& M_{11}^{(1,1)}=-\mathcal{K}_{(\pi \pi \mid \pi \pi)}^{(1,1)}\left[\Omega_{\pi \pi}^{P} / 2\right], \quad M_{12}^{(1,1)}=-\mathcal{K}_{(\pi \pi \mid K K)}^{(1,1)}\left[\Omega_{K K}^{P} / 2\right] \\
& M_{21}^{(1,1)}=-\mathcal{K}_{(\pi \pi \mid K K)}^{(1,1)}\left[\Omega_{\pi \pi}^{P} / 2\right], \quad M_{22}^{(1,1)}=-\mathcal{K}_{(K K \mid K K)}^{(1,1)}\left[\Omega_{K K}^{P} / 2\right] .
\end{aligned}
$$

$$
\begin{array}{ll}
M_{11}^{(1,1 / 2)}=-\mathcal{K}_{(\pi K \mid \pi K)}^{(1,1 / 2)}\left[\Omega_{\pi K}^{P}\right], & M_{12}^{(1,1 / 2)}=-\mathcal{K}_{(\pi K \mid K 8)}^{(1,1 / 2)}\left[\Omega_{K 8}^{P}\right], \\
M_{21}^{(1,1 / 2)}=-\mathcal{K}_{(\pi K \mid K 8)}^{(1,1 / 2)}\left[\Omega_{\pi K}^{P}\right], & M_{22}^{(1,1 / 2)}=-\mathcal{K}_{(K 8 \mid K 8)}^{(1,1 / 2)}\left[\Omega_{K 8}^{P}\right] .
\end{array}
$$

$$
M^{(1,0)}=-\mathcal{K}_{(K K \mid K K)}^{(1,0)}\left[\Omega_{K K}^{P} / 2\right],
$$

and

$$
\begin{aligned}
& M_{11}^{(0,1)}=-\mathcal{K}_{(\pi 8 \mid \pi 8)}^{(0,1)}\left[\Omega_{\pi 8}^{S} / 2\right], \quad M_{12}^{(0,1)}=-\mathcal{K}_{(\pi 8 \mid K K)}^{(0,1)}\left[\Omega_{K K}^{S} / 2\right], \\
& M_{21}^{(0,1)}=-\mathcal{K}_{(\pi 8 \mid K K)}^{(0,1)}\left[\Omega_{\pi 8}^{S} / 2\right], \quad M_{22}^{(0,1)}=-\mathcal{K}_{(K K \mid K K)}^{(0,1)}\left[\Omega_{K K}^{S} / 2\right] .
\end{aligned}
$$

$$
\begin{array}{ll}
M_{11}^{(0,1 / 2)}=-\mathcal{K}_{(\pi K \mid \pi K)}^{(1,1 / 2)}\left[\Omega_{\pi K}^{S}\right], & M_{12}^{(0,1 / 2)}=-\mathcal{K}_{(\pi K \mid K 8)}^{(1,1 / 2)}\left[\Omega_{K 8}^{S}\right], \\
M_{21}^{(0,1 / 2)}=-\mathcal{K}_{(\pi K \mid K 8)}^{(1,1 / 2)}\left[\Omega_{\pi K}^{S}\right], & M_{22}^{(0,1 / 2)}=-\mathcal{K}_{(K 8 \mid K 8)}^{(1,1 / 2)}\left[\Omega_{K 8}^{S}\right] .
\end{array}
$$

$$
\begin{array}{ll}
M_{11}^{(0,0)}=-\mathcal{K}_{(\pi \pi \mid \pi \pi)}^{(0,0)}\left[\Omega_{\pi \pi}^{S} / 2\right], & M_{12}^{(0,0)}=-\mathcal{K}_{(\pi \pi \mid K K)}^{(0,0)}\left[\Omega_{K K}^{S} / 2\right], \\
M_{13}^{(0,0)}=-\mathcal{K}_{(\pi \pi \mid 88)}^{(0,0)}\left[\Omega_{88}^{S} / 2\right], & M_{21}^{(0,0)}=-\mathcal{K}_{(\pi \pi \mid K K)}^{(0,0)}\left[\Omega_{\pi \pi}^{S} / 2\right], \\
M_{22}^{(0,0)}=-\mathcal{K}_{(K K \mid K K)}^{(0,0)}\left[\Omega_{K K}^{S} / 2\right], & M_{23}^{(0,0)}=-\mathcal{K}_{(K K \mid 88)}^{(0,0)}\left[\Omega_{88}^{S} / 2\right], \\
M_{31}^{(0,0)}=-\mathcal{K}_{(\pi \pi \mid 88)}^{(0,0)}\left[\Omega_{\pi \pi}^{S} / 2\right], & M_{32}^{(0,0)}=-\mathcal{K}_{(K K \mid 88)}^{(0,0)}\left[\Omega_{K K}^{S} / 2\right], \\
M_{33}^{(0,0)}=-\mathcal{K}_{(88 \mid 88)}^{(0,0)}\left[\Omega_{88}^{S} / 2\right] . & (\mathrm{C} 6)
\end{array}
$$

The factor $1 / 2$ accounts for the symmetry of intermediate states. It is also present in the functions $M_{11}^{(0,1)}$ and $M_{21}^{(0,1)}$ because we use symmetrized $\pi 8$ intermediate states.

As shown in Eqs. (6) and (7), the summation of the geometric series yields scattering amplitudes based on denominators given schematically by $D=1-($ loop $\times \mathcal{K})$,

$$
D^{(1,1)}=\left[1-M_{11}^{(1,1)}\right]\left[1-M_{22}^{(1,1)}\right]-M_{12}^{(1,1)} M_{21}^{(1,1)},
$$

$$
\begin{aligned}
& D^{(1,1 / 2)}= {\left[1-M_{11}^{(1,1 / 2)}\right]\left[1-M_{22}^{(1,1 / 2)}\right]-M_{12}^{(1,1 / 2)} M_{21}^{(1,1 / 2)}, } \\
& D^{(0,1)}=\left[1-M_{11}^{(0,1)}\right]\left[1-M_{22}^{(0,1)}\right]-M_{12}^{(0,1)} M_{21}^{(0,1)}, \quad(\mathrm{C} 9) \\
& D^{(0,1 / 2)}=\left[1-M_{11}^{(0,1 / 2)}\right]\left[1-M_{22}^{(0,1 / 2)}\right]-M_{12}^{(0,1 / 2)} M_{21}^{(0,1 / 2)}, \\
& D^{(0,0)}=\left[1-M_{11}^{(0,0)}\right]\left[1-M_{22}^{(0,0)}\right]\left[1-M_{33}^{(0,0)}\right] \\
&-\left[1-M_{11}^{(0,0)}\right] M_{23}^{(0,0)} M_{32}^{(0,0)} \\
&-\left[1-M_{22}^{(0,0)}\right] M_{13}^{(0,0)} M_{31}^{(0,0)}-\left[1-M_{33}^{(0,0)}\right] M_{12}^{(0,0)} M_{21}^{(0,0)} \\
&-M_{12}^{(0,0)} M_{23}^{(0,0)} M_{31}^{(0,0)}-M_{21}^{(0,0)} M_{13}^{(0,0)} M_{32}^{(0,0)} . \quad(\mathrm{C} 11)
\end{aligned}
$$

The scattering amplitudes for the process $P_{k} P_{\ell} \rightarrow P_{a} P_{b}$ in the various channels are given as follows.

(1) Vector sector:

- isospin $I=1$ :

$$
\begin{aligned}
A_{(\pi \pi \mid a b)}^{(1,1)}= & \frac{1}{D^{(1,1)}}\left\{\left[1-M_{22}^{(1,1)}\right] \mathcal{K}_{(\pi \pi \mid a b)}^{(1,1)}\right. \\
& \left.+M_{12}^{(1,1)} \mathcal{K}_{(K K \mid a b)}^{(1,1)}\right\}(t-u), \\
A_{(K K \mid a b)}^{(1,1)}= & \frac{1}{D^{(1,1)}}\left\{M_{21}^{(1,1)} \mathcal{K}_{(\pi \pi \mid a b)}^{(1,1)}\right. \\
& \left.+\left[1-M_{11}^{(1,1)}\right] \mathcal{K}_{(K K \mid a b)}^{(1,1)}\right\}(t-u) .
\end{aligned}
$$

- isospin $I=1 / 2$ :

$$
\begin{aligned}
& A_{(\pi K \mid a b)}^{(1,1 / 2)}= \frac{1}{D^{(1,1 / 2)}}\left\{\left[1-M_{22}^{(1,1 / 2)}\right] \mathcal{K}_{(\pi K \mid a b)}^{(1,1 / 2)}\right. \\
&\left.+M_{12}^{(1,1 / 2)} \mathcal{K}_{(K 8 \mid a b)}^{(1,1 / 2)}\right\} \\
& \times\left[2 t+s-2\left(M_{\pi}^{2}+M_{K}^{2}\right)+\frac{\left(M_{\pi}^{2}-M_{K}^{2}\right)^{2}}{s}\right], \\
&(\mathrm{C} 14) \\
& A_{(K 8 \mid a b)}^{(1,1 / 2)}= \frac{1}{D^{(1,1 / 2)}}\left\{M_{21}^{(1,1 / 2)} \mathcal{K}_{(\pi K \mid a b)}^{(1,1 / 2)}\right. \\
&\left.+\left[1-M_{11}^{(1,1 / 2)}\right] \mathcal{K}_{(K 8 \mid a b)}^{(1,1 / 2)}\right\} \\
& \times\left[2 t+s-2\left(M_{\pi}^{2}+M_{K}^{2}\right)+\frac{\left(M_{\pi}^{2}-M_{K}^{2}\right)^{2}}{s}\right] .
\end{aligned}
$$

- isospin $I=0$ :

$$
A_{(K K \mid a b)}^{(1,0)}=\frac{1}{D^{(1,0)}} \mathcal{K}_{(K K \mid a b)}^{(1,0)}(t-u) .
$$


(2) Scalar sector:

- isospin $I=1$ :

$$
\begin{aligned}
A_{(\pi 8 \mid a b)}^{(0,1)}= & \frac{1}{D^{(0,1)}}\left\{\left[1-M_{22}^{(0,1)}\right] \mathcal{K}_{(\pi 8 \mid a b)}^{(0,1)}\right. \\
& \left.+M_{12}^{(0,1)} \mathcal{K}_{(K K \mid a b)}^{(0,1)}\right\} \\
A_{(K K \mid a b)}^{(0,1)}= & \frac{1}{D^{(0,1)}}\left\{M_{21}^{(0,1)} \mathcal{K}_{(\pi 8 \mid a b)}^{(0,1)}\right. \\
& \left.+\left[1-M_{11}^{(0,1)}\right] \mathcal{K}_{(K K \mid a b)}^{(0,1)}\right\}
\end{aligned}
$$

- isospin $I=1 / 2$ :

$$
\begin{aligned}
A_{(\pi K \mid a b)}^{(0,1 / 2)}= & \frac{1}{D^{(0,1 / 2)}}\left\{\left[1-M_{22}^{(0,1 / 2)}\right] \mathcal{K}_{(\pi K \mid a b)}^{(0,1 / 2)}\right. \\
& \left.+M_{12}^{(1,1 / 2)} \mathcal{K}_{(K 8 \mid a b)}^{(0,1 / 2)}\right\}, \\
A_{(K 8 \mid a b)}^{(0,1 / 2)}= & \frac{1}{D^{(0,1 / 2)}}\left\{M_{21}^{(0,1 / 2)} \mathcal{K}_{(\pi K \mid a b)}^{(0,1 / 2)}\right. \\
& \left.+\left[1-M_{11}^{(0,1 / 2)}\right] \mathcal{K}_{(K 8 \mid a b)}^{(0,1 / 2)}\right\} .
\end{aligned}
$$

$$
\begin{aligned}
A_{(\pi \pi \mid a b)}^{(0,0)}= & \frac{1}{D^{(0,0)}}\left\{\left[\left(1-M_{22}^{(0,0)}\right)\left(1-M_{33}^{(0,0)}\right)-M_{23}^{(0,0)} M_{32}^{(0,0)}\right] \mathcal{K}_{(\pi \pi \mid a b)}^{(0,0)}+\left[M_{12}^{(0,0)}\left(1-M_{33}^{(0,0)}\right)+M_{13}^{(0,0)} M_{32}^{(0,0)}\right] \mathcal{K}_{(K K \mid a b)}^{(0,0)}\right. \\
& \left.+\left[M_{13}^{(0,0)}\left(1-M_{22}^{(0,0)}\right)+M_{12}^{(0,0)} M_{23}^{(0,0)}\right] \mathcal{K}_{(88 \mid a b)}^{(0,0)}\right\}, \\
A_{(K K \mid a b)}^{(0,0)}= & \frac{1}{D^{(0,0)}}\left\{\left[M_{21}^{(0,0)}\left(1-M_{33}^{(0,0)}\right)+M_{23}^{(0,0)} M_{31}^{(0,0)}\right] \mathcal{K}_{(\pi \pi \mid a b)}^{(0,0)}+\left[\left(1-M_{11}^{(0,0)}\right)\left(1-M_{33}^{(0,0)}\right)-M_{13}^{(0,0)} M_{31}^{(0,0)}\right) \mathcal{K}_{(K K \mid a b)}^{(0,0)}\right. \\
& \left.+\left[M_{23}^{(0,0)}\left(1-M_{11}^{(0,0)}\right)+M_{13}^{(0,0)} M_{21}^{(0,0)}\right] \mathcal{K}_{(88 \mid a b)}^{(0,0)}\right\}, \\
A_{(88 \mid a b)}^{(0,0)}= & \frac{1}{D^{(0,0)}}\left\{\left[M_{31}^{(0,0)}\left(1-M_{22}^{(0,0)}\right)+M_{21}^{(0,0)} M_{32}^{(0,0)}\right] \mathcal{K}_{(\pi \pi \mid a b)}^{(0,0)}+\left[M_{32}^{(0,0)}\left(1-M_{11}^{(0,0)}\right)+M_{12}^{(0,0)} M_{31}^{(0,0)}\right] \mathcal{K}_{(K K \mid a b)}^{(0,0)}\right. \\
& \left.+\left[\left(1-M_{11}^{(0,0)}\right)\left[1-M_{22}^{(0,0)}\right)-M_{12}^{(0,0)} M_{21}^{(0,0)}\right] \mathcal{K}_{(88 \mid a b)}^{(0,0)}\right\} .
\end{aligned}
$$

\section{APPENDIX D: $\pi \pi$ PHASE SHIFTS}

Most examples discussed in the main text refer to $\pi \pi$ scattering, and the partial-wave expansion of the amplitude for isospin channel $I$ reads

$$
A_{(\pi \pi \mid \pi \pi)}^{I}=\frac{32 \pi}{\rho} \sum_{J=0}^{\infty}(2 J+1) P_{J}(\cos \theta) f_{(\pi \pi \mid \pi \pi)}^{(J, I)}(s),
$$

where $f_{(\pi \pi \mid \pi \pi)}^{(J, I)}$ is the nonrelativistic scattering amplitude and $\rho=\sqrt{\left(s-4 M_{\pi}^{2}\right) / s}$. Our amplitudes are written as

$$
A_{(\pi \pi \mid \pi \pi)}^{I}=A_{(\pi \pi \mid \pi \pi)}^{(0, I)}+A_{(\pi \pi \mid \pi \pi)}^{(1, I)}+\cdots .
$$

In the c.m. frame, one has $(t-u)=\left(s-4 M_{\pi}^{2}\right) \cos \theta$ and

$$
\begin{aligned}
A_{(\pi \pi \mid \pi \pi)}^{I} & =A_{(\pi \pi \mid \pi \pi)}^{(0, I)}+\left[\left(s-4 M_{\pi}^{2}\right) \cos \theta\right] A_{(\pi \pi \mid \pi \pi)}^{(1, I)}+\cdots \\
& =\frac{32 \pi}{\rho}\left[f_{(\pi \pi \mid \pi \pi)}^{(0, I)}(s)+3 \cos \theta f_{(\pi \pi \mid \pi \pi)}^{(1, I)}(s)+\cdots\right],
\end{aligned}
$$

$$
\begin{gathered}
f_{(\pi \pi \mid \pi \pi)}^{(0,0)}=\frac{\sqrt{s-4 M_{\pi}^{2}}}{32 \pi \sqrt{s}} A_{(\pi \pi \mid \pi \pi)}^{(0,0)}, \\
f_{(\pi \pi \mid \pi \pi)}^{(1,1)}=\frac{\left(s-4 M_{\pi}^{2}\right)^{3 / 2}}{96 \pi \sqrt{s}} A_{(\pi \pi \mid \pi \pi)}^{(1,1)} .
\end{gathered}
$$

From now on, we drop all subscripts and superscripts and express the amplitude $f$ in terms of phase shifts $\delta$ and inelasticity parameters $\eta$ as [15]

$$
f=\frac{1}{2 i}\left[\eta e^{2 i \delta}-1\right] .
$$

In order to obtain $\delta$ and $\eta$ from the $A_{\pi \pi \mid \pi \pi}^{(J, I)}$, one writes $f=a+i b$, with $a=\operatorname{Re}[f]$ and $b=\operatorname{Im}[f]$ and Eq. (D6) yields

$$
1+2 i f=[1-2 b]+2 i a=\eta[\cos 2 \delta+i \sin 2 \delta] .
$$

Thus,

$$
\eta=\sqrt{[1-2 b]^{2}+4 a^{2}},
$$

with 


$$
\delta=\tan ^{-1}\left[\frac{2 a}{1+\eta-2 b}\right] .
$$

The alternative form

$$
\delta=\frac{1}{2} \tan ^{-1}\left[\frac{\sin 2 \delta}{\cos 2 \delta}\right]
$$

$$
\sin 2 \delta=\frac{2 a}{\eta}, \quad \cos 2 \delta=\frac{1-2 b}{\eta}
$$

is more convenient in numerical calculations because, as $\eta>0$, the signs of $\sin 2 \delta$ and $\cos 2 \delta$ in Eq. (D11) are well defined and the quadrant assignment of $2 \delta$ is unambiguous. This yields continuous results in the interval $0 \leq \delta \leq \pi$.
[1] E. M. Aitala et al. (E791 Collaboration), Phys. Rev. Lett. 86, 770 (2001).

[2] E. M. Aitala et al. (E791 Collaboration), Phys. Rev. Lett. 89, 121801 (2002); J. M. Link et al. (FOCUS Collaboration), Phys. Lett. B 681, 14 (2009).

[3] I. Bediaga, T. Frederico, and O. Lourenço, Phys. Rev. D 89, 094013 (2014); J. H. A. Nogueira, I. Bediaga, A. B. R. Cavalcante, T. Frederico, and O. Lourenço, Phys. Rev. D 92, 054010 (2015); I. Bediaga, T. Frederico, and P. C. Magalhães, Phys. Lett. B 806, 135490 (2020).

[4] D. Aston et al., Nucl. Phys. B296, 493 (1988).

[5] P. C. Magalhães, M. R. Robilotta, K. S. F. F. Guimarães, T. Frederico, W. S. de Paula, I. Bediaga, A. C. dos Reis, and C. M. Maekawa, Phys. Rev. D 84, 094001 (2011); K. S. F. F. Guimarães, W. de Paula, I. Bediaga, A. Delfino, T. Frederico, A. C. dos Reis, and L. Tomio, Nucl. Phys. B, Proc. Suppl. 199, 341 (2010).

[6] P. C. Magalhães and M. R. Robilotta, Phys. Rev. D 92, 094005 (2015).

[7] F. Niecknig and B. Kubis, J. High Energy Phys. 10 (2015) 142.

[8] S. X. Nakamura, Phys. Rev. D 93, 014005 (2016).

[9] G. Ecker and R. Unterdorfer, Eur. Phys. J. C 24, 535 (2002); Nucl. Phys. B, Proc. Suppl. 121, 175 (2003).

[10] R. Aoude, P. Magalhães, A. Dos Reis, and M. Robilotta, Phys. Rev. D 98, 056021 (2018).

[11] I. J. R. Aitchison, arXiv:1507.02697; J. H. A. Nogueira, arXiv:1605.03889; J. Pelaez, Phys. Rep. 658, 1 (2016).

[12] P. C. Magalhães and M. R. Robilotta, Phys. Rev. D 90, 014043 (2014).

[13] S. Ropertz, C. Hanhart, and B. Kubis, Eur. Phys. J. C 78, 1000 (2018).

[14] J. A. Oller and E. Oset, Phys. Rev. D 60, 074023 (1999); Nucl. Phys. A620, 465 (1997); A652, 407(E) (1999); J. R. Pelaez, J. A. Oller, and E. Oset, Nucl. Phys. A675, 92C (2000); K. P. Khemchandani, A. Martinez Torres, H. Nagahiro, and A. Hosaka, Phys. Rev. D 88, 114016 (2013).

[15] B. Hyams et al., Nucl. Phys. B64, 134 (1973).

[16] M. Albaladejo and B. Moussallam, Eur. Phys. J. C 77, 508 (2017).

[17] J. Pelaez, A. Rodas, and J. Ruiz De Elvira, Eur. Phys. J. C 79, 1008 (2019).

[18] R. Aaij et al. (LHCb Collaboration), J. High Energy Phys. 04 (2019) 063.

[19] V. Anisovich and A. Sarantsev, Eur. Phys. J. A 16, 229 (2003).
[20] L-L. Chau, Phys. Rep. 95, 1 (1983).

[21] D. Boito, J.-P. Dedonder, B. El-Bennich, R. Escribano, R. Kaminski, L. Lesniak, and B. Loiseau, Phys. Rev. D 96, 113003 (2017); R. Klein, T. Mannel, J. Virto, and K. K. Vos, J. High Energy Phys. 10 (2017) 117.

[22] G. Burdman and J. F. Donoghue, Phys. Lett. B 280, 287 (1992); M. B. Wise, Phys. Rev. D 45, R2188 (1992); R. Casalbuoni, A. Deandrea, N. Di Bartolomeo, R. Gatto, F. Feruglio, and G. Nardulli, Phys. Rep. 281, 145 (1997).

[23] R. A. Briceno, J. J. Dudek, and R. D. Young, Rev. Mod. Phys. 90, 025001 (2018).

[24] S. Weinberg, Physica (Amsterdam) 96A, 327 (1979).

[25] J. Gasser and H. Leutwyler, Ann. Phys. (N.Y.) 158, 142 (1984).

[26] J. Gasser and H. Leutwyler, Nucl. Phys. B250, 465 (1985).

[27] G. Ecker, J. Gasser, A. Pich, and E. De Rafael, Nucl. Phys. B321, 311 (1989).

[28] L. Y. Dai and M. R. Pennington, Phys. Rev. D 90, 036004 (2014).

[29] S. Ropertz, C. Hanhart, and B. Kubis, Eur. Phys. J. C 78, 1000 (2018).

[30] B. Kubis, EPJ Web Conf. 218, 0300 (2019); I. Caprini, G. Colangelo, and H. Leutwyler, Eur. Phys. J. C 72, 1860 (2012); B. Ananthanarayan, G. Colangelo, J. Gasser, and H. Leutwyler, Phys. Rep. 353, 207 (2001); P. Buettiker, S. Descotes-Genon, and B. Moussallam, Eur. Phys. J. C 33, 409 (2004); S. Descotes-Genon and B. Moussallam, Eur. Phys. J. C 48, 553 (2006); R. Garcia-Martin, R. Kaminski, J. Pelaez, J. Ruiz de Elvira, and F. Yndurain, Phys. Rev. D 83, 074004 (2011).

[31] M. Albaladejo and B. Moussallam, Eur. Phys. J. C 77, 508 (2017).

[32] R. A. Briceño, M. T. Hansen, and S. R. Sharpe, Phys. Rev. D 95, 074510 (2017); M. Mai and M. Döring, Eur. Phys. J. A 53, 240 (2017); A. Jackura et al. (JPAC Collaboration), Eur. Phys. J. C 79, 56 (2019); A. W. Jackura, S. M. Dawid, C. Fernández-Ramírez, V. Mathieu, M. Mikhasenko, A. Pilloni, S. R. Sharpe, and A. P. Szczepaniak, Phys. Rev. D 100, 034508 (2019); R. A. Briceño, M. T. Hansen, S. R. Sharpe, and A. P. Szczepaniak, Phys. Rev. D 100, 054508 (2019).

[33] P. A. Zyla et al. (Particle Data Group), Prog. Theor. Exp. Phys. 2020, 083C01 (2020).

[34] G. Gounaris and J. Sakurai, Phys. Rev. Lett. 21, 244 (1968). 